\title{
Getting Started in Synthesis: A Tabular Guide to Selected Monofunctional Fluorous Compounds
}

\author{
József Rábai
}

9.1

Introduction

The first steps when beginning a fluorous project are to purchase the necessary fluorous starting materials and synthesize the target molecules. The vendors of fluorous solvents listed in Table 3-1 also provide a number of fluorous building blocks, as does Fluorous Technologies Inc. (FTI, http://fluorous.com/index2.html). This chapter, augmented by the references to the table of partition coefficients in Chapter 6 (Table 6-1), is intended as a guide to the synthesis of simple fluorous molecules. It is by no means comprehensive, but does provide many useful leads for researchers just beginning in this field.

Looking first backwards to Table 6-1, it is easy to visually scan various families of functional groups and locate lead references. However, if the partition coefficient has not been measured, the molecule is not included. Furthermore, superior syntheses may have been developed after the initial literature report.

Recognizing this gap, this chapter summarizes a wide range of monofunctional "heavy" fluorous compounds and selected "light" fluorous compounds in a tabular form. In most cases, abbreviated synthetic details are indicated (" $\Rightarrow$ " symbol), followed by the literature reference. In some cases, particularly useful reactions are indicated ("•+" symbol). More complex target molecules can then be assembled from perfluoroalkyl- and organic groups of appropriate topologies, sometimes inserting "insulator" groups between these constituents (cf. Chapters 4 and 5).

The latter strategy or modular synthesis calls for an "F-tool-kit" suitable for the most diverse applications. At one extreme, various target $F$-entities with linear perfluoroalkyl groups exhibit good organic solubilities under reaction conditions at higher temperatures, but quantitatively precipitate at lower temperatures (thermomorphism). At the other extreme $F$ derivatives with flexible and branched perfluoropolyether type substituents could have rather wide fluid temperature ranges (high-tech lubricants).

Syntheses involving highly fluorinated compounds will certainly provide simultaneous feelings of frustration and pleasure for those so engaged. This derives from differences in reactivity and solubility patterns from those experienced in traditional organic chemistry. The high electronegativity of fluorine atoms and perfluoroalkyl-groups strongly affect reac-

Handbook of Fluorous Chemistry. Edited by John A. Gladysz, Dennis P. Curran, István T. Horvath Copyright (C) 2004 WILEY-VCH Verlag GmbH \& Co. KGaA, Weinheim ISBN: 3-527-30617-X 
tion centers, unless appropriately positioned (cf. Chapter 5). Moreover, macroscopic properties, such as solubility, fluorophilicity, volatility, melting and boiling point, transition enthalpies, etc. are all governed by the composition and structure of the molecules. The higher their fluorous character, the more unique properties will be expressed.

The trifluoromethyl group, which can be regarded as the shortest F-ponytail, appears in the Beilstein database with a frequency one order of magnitude greater than all longer $\mathrm{R}_{\mathrm{fn}}$ segments combined $\left(\mathrm{R}_{\mathrm{f} 1} \mathrm{GH}^{*} / \mathrm{R}_{\mathrm{f} 2-24} \mathrm{GH}^{*}=205000: 24000\right)$. Hence, some prototype chemistries known only for $\mathrm{CF}_{3}$-compounds are also displayed in the Tables. In the first series of Tables, molecules without hydrogen atoms are collected. In the second, hydrogen atoms of all descriptions are allowed.

\section{Series I [no hydrogen included]}

9.1.1 Perfluoroalkanes, perfluoroalkenes and perfluoroalkynes

9.1.2 Perfluoroalkyl halides and related compounds

9.1.3 Perfluoroethers, perfluoroalkanones and perfluoroalkanecarboxylic acid halides

9.1.4 Perfluoroalkylsulfides and disulfides, perfluoroalkanesulfonyl halides and anhydrides

9.1.5 Tris(perfluoroalkyl)amines, perfluoroazomethines, perfluoroalkanecarbonitriles, tris(perfluoroalkyl)triazines and perfluoroalkyl isocyanates

9.1.6 Perfluoroalkylmagnesium-, zinc-, copper(I)-, lithium-, and caesium intermediates.

Tab. 9.1-1. Perfluoroalkanes, perfluoroalkenes and perfluoroalkynes

\begin{tabular}{|c|c|c|}
\hline Compound $[C, F]$ & Remarks (" $\Rightarrow$ "for synthesis of; “•+" for reaction of; etc.) & Ref. \\
\hline $\mathrm{C}_{n} \mathrm{~F}_{2 n+2}$, cyclo- $\mathrm{C}_{n} \mathrm{~F}_{2 n}$, etc. & $\begin{array}{l}\Rightarrow \bullet+\text { Disclosure of a "Secret World": Properties of } \\
\text { Fluorocarbons }\end{array}$ & 1 \\
\hline Perfluoroalkanes & $\begin{array}{l}\Rightarrow \bullet+\text { Synthesis and Chemistry of Perfluoroalkanes. } \\
\text { A Review }\end{array}$ & 2 \\
\hline $\mathrm{CF}_{3} \mathrm{CF}_{2} \mathrm{CF}=\mathrm{C}\left(\mathrm{CF}_{3}\right)_{2}$ & $\begin{array}{l}\bullet+\mathrm{KF}+\mathrm{ROCH}_{2} \mathrm{Cl} / \mathrm{PTC}, \text { rt. } \rightarrow \mathrm{ROCH}_{2} \mathrm{C}\left(\mathrm{CF}_{3}\right)_{2} \mathrm{CF}_{2} \mathrm{CF}_{2} \mathrm{CF}_{3} \\
\quad(82 \%)\end{array}$ & 3 \\
\hline $\mathrm{CF}_{3}\left(\mathrm{CF}_{2}\right)_{3} \mathrm{CF}=\mathrm{CF}_{2}$ & $\begin{aligned} \Rightarrow & \mathrm{R}_{\mathrm{f} 6} \mathrm{CO}_{2} \mathrm{Na} / \text { heating } \rightarrow \mathrm{R}_{\mathrm{f} 4} \mathrm{CF}=\mathrm{CF}_{2}+\text { isomers, in a } \\
& 73: 27 \text { ratio }\end{aligned}$ & 4 \\
\hline $\mathrm{R}_{\mathrm{fn}} \mathrm{CF}=\mathrm{CF}_{2}, n=1,2,3,5,7$ & $\Rightarrow$ Prepared by the pyrolysis of the salts of $\mathrm{R}_{\mathrm{f} n+2} \mathrm{CO}_{2} \mathrm{H}$ 's & 5 \\
\hline$\left(\mathrm{CF}_{3}\right)_{2} \mathrm{C}=\mathrm{CF}_{2}$ & $\begin{array}{l}\Rightarrow \text { cyclo }-\mathrm{C}_{4} \mathrm{~F}_{8} / 25 \mathrm{~s} \text { at } 710-730^{\circ} \mathrm{C} / \rightarrow \text { yield: } 45 \% \\
\quad \mathrm{bp}=6-9{ }^{\circ} \mathrm{C}\end{array}$ & 6 \\
\hline $\begin{aligned} \mathrm{R}_{\mathrm{fn}} \mathrm{C} \equiv \mathrm{CR}_{\mathrm{f} m}, n=2,4,8 \\
\quad m \\
\quad=4,6,8\end{aligned}$ & $\Rightarrow \mathrm{R}_{\mathrm{fn}} \mathrm{C} \equiv \mathrm{CH}+\mathrm{R}_{\mathrm{fm}} \mathrm{I} / 220^{\circ} \mathrm{C} \rightarrow \mathrm{R}_{\mathrm{fn}} \mathrm{CI}=\mathrm{CHR}_{\mathrm{fm}} /$ base-PTC $\rightarrow$ & 7 \\
\hline
\end{tabular}


Tab. 9.1-2. Perfluoroalkyl halides and related compounds

\begin{tabular}{|c|c|c|}
\hline Compound $[C, F, X]$ & Remarks (“ $\Rightarrow$ " for synthesis of; “•+" for reaction of; etc.) & Ref. \\
\hline $\mathrm{R}_{\mathrm{f} n} \mathrm{I}, n=1,3$ & $\Rightarrow \mathrm{R}_{\mathrm{fn}} \mathrm{CO}_{2} \mathrm{Na}+\mathrm{I}_{2} / \mathrm{DMF}$, reflux $\rightarrow$ yield: $70-80 \%$ & 8 \\
\hline $\mathrm{R}_{\mathrm{f} n} \mathrm{I}, n=1-12$ & $\Rightarrow$ Ref. 9. Uses and reactions of: Ref. 10 & 9,10 \\
\hline$\left(\mathrm{CF}_{3}\right)_{2} \mathrm{CF}\left(\mathrm{CF}_{2} \mathrm{CF}_{2}\right)_{n} \mathrm{I}$ & $\begin{aligned} \Rightarrow & \left(\mathrm{CF}_{3}\right)_{2} \mathrm{CFI}+\mathrm{CF}_{2}=\mathrm{CF}_{2} / 175^{\circ} \mathrm{C} \rightarrow \text { mix of telomers; } \\
& n=1>n=2-4\end{aligned}$ & 11 \\
\hline $\mathrm{Cl}\left(\mathrm{CF}_{2}\right)_{n} \mathrm{I}, n=2,4,6$ & - $+\mathrm{CH}_{2}=\mathrm{CHR} /$ acid treated Fe catalyst $\rightarrow \mathrm{Cl}\left(\mathrm{CF}_{2}\right)_{n} \mathrm{CH}_{2} \mathrm{CH}(\mathrm{I}) \mathrm{R}$ & 12 \\
\hline $\mathrm{C}_{8} \mathrm{~F}_{17} \mathrm{Br}$ & $\Rightarrow \mathrm{C}_{8} \mathrm{~F}_{17} \mathrm{H}+\mathrm{CBr}_{4} / 300^{\circ} \mathrm{C}, 24 \mathrm{~h} \rightarrow$ yield: $40 \%$ & 13 \\
\hline $\mathrm{C}_{8} \mathrm{~F}_{17} \mathrm{Cl}$ & $\Rightarrow \mathrm{C}_{8} \mathrm{~F}_{17} \mathrm{H}+\mathrm{CCl}_{4} / 350^{\circ} \mathrm{C}, 24 \mathrm{~h} \rightarrow$ yield: $31 \%$ & 13 \\
\hline $\mathrm{X}\left(\mathrm{CF}_{2}\right)_{n} \mathrm{Cl}$ & $\begin{array}{l}\bullet+\mathrm{CH}_{2}=\mathrm{CHR} /\left(\mathrm{NH}_{4}\right)_{2} \mathrm{~S}_{2} \mathrm{O}_{8}-\mathrm{HCO}_{2} \mathrm{Na} \rightarrow \mathrm{X}\left(\mathrm{CF}_{2}\right)_{n}\left(\mathrm{CH}_{2}\right)_{2} \mathrm{R} \\
\quad(70-80 \%)\end{array}$ & 14 \\
\hline $\mathrm{R}_{\mathrm{f} 6} \mathrm{CCl}_{3}$ & $\Rightarrow \mathrm{R}_{\mathrm{f} 6} \mathrm{I}+\mathrm{CCl}_{4} / \mathrm{Zn}, \mathrm{EtCO}_{2} \mathrm{H}, \mathrm{CH}_{2} \mathrm{Cl}_{2}, 2 \mathrm{~h} \rightarrow$ yield: $51 \%$ & 15 \\
\hline $\mathrm{R}_{\mathrm{f} 9} \mathrm{CCl}_{3}$ & $\Rightarrow \mathrm{R}_{\mathrm{f} 10} \mathrm{I}+\mathrm{AlCl}_{3} / \sim 115^{\circ} \mathrm{C}, 28 \mathrm{~h} \rightarrow$ yield: $55 \%$ & 16 \\
\hline$\left(\mathrm{CF}_{3}\right)_{2} \mathrm{CFO}\left(\mathrm{CF}_{2}\right)_{3} \mathrm{CCl}_{3}$ & $\Rightarrow\left(\mathrm{CF}_{3}\right)_{2} \mathrm{CFO}\left(\mathrm{CF}_{2}\right)_{4} \mathrm{I}+\mathrm{AlCl}_{3} / 100^{\circ} \mathrm{C}, 63 \mathrm{~h} \rightarrow$ yield: $43 \%$ & 16 \\
\hline $\mathrm{CCl}_{3}\left(\mathrm{CF}_{2}\right)_{4} \mathrm{~N}=\mathrm{N}\left(\mathrm{CF}_{2}\right)_{4} \mathrm{CCl}_{3}$ & $\begin{array}{l}\bullet+\mathrm{SO}_{3} / \mathrm{H}_{2} \mathrm{SO}_{4} / \mathrm{Hg}^{2+} \rightarrow \mathrm{HO}_{2} \mathrm{C}\left(\mathrm{CF}_{2}\right)_{4} \mathrm{~N}=\mathrm{N}\left(\mathrm{CF}_{2}\right)_{4} \mathrm{CO}_{2} \mathrm{H}, \\
\quad \text { yield: } 95 \%\end{array}$ & 17 \\
\hline$\left(\mathrm{CF}_{3} \mathrm{CF}_{2}\right)_{2} \mathrm{NCF}_{2} \mathrm{CF}_{2} \mathrm{I}$ & $\Rightarrow\left(\mathrm{CF}_{3} \mathrm{CF}_{2}\right)_{2} \mathrm{NCF}_{2} \mathrm{CF}_{2} \mathrm{COF} / \mathrm{LiI}, 180{ }^{\circ} \mathrm{C}, 18 \mathrm{~h} \rightarrow$ yield: $72 \%$ & 18 \\
\hline $\mathrm{CF}_{3}\left(\mathrm{CF}_{2}\right)_{7} \mathrm{OCF}_{2} \mathrm{CF}_{2} \mathrm{I}$ & $\Rightarrow \mathrm{R}_{\mathrm{f} 7} \mathrm{COF}+\mathrm{CF}_{2}=\mathrm{CF}_{2} / \mathrm{KF}$, diglyme, $-196{ }^{\circ} \mathrm{C}, 7 \mathrm{~d} \rightarrow$ & 19 \\
\hline $\mathrm{C}_{3} \mathrm{~F}_{7} \mathrm{OCF}\left(\mathrm{CF}_{3}\right) \mathrm{CF}_{2} \mathrm{OCFICF}_{3}$ & 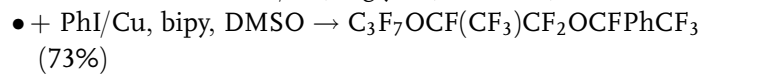 & 20 \\
\hline$\left(\mathrm{CF}_{3}\right)_{2} \mathrm{CFOCF}_{2} \mathrm{CF}_{2} \mathrm{I}$ & $\begin{aligned} \Rightarrow & \left(\mathrm{CF}_{3}\right)_{2} \mathrm{CFOK}+\mathrm{CF}_{2}=\mathrm{CF}_{2}+\mathrm{I}_{2} \text { or } \mathrm{ICl} / \mathrm{CH}_{3} \mathrm{CN} \rightarrow \mathrm{bp}= \\
& 86-87^{\circ} \mathrm{C}\end{aligned}$ & 21 \\
\hline $\mathrm{F}_{5} \mathrm{SCF}_{2} \mathrm{I}$ & $\Rightarrow \mathrm{F}_{5} \mathrm{SCF}_{2} \mathrm{CO}_{2} \mathrm{Ag}+\mathrm{I}_{2} /$ heating $\rightarrow$ yield: $54 \%$ & 22 \\
\hline $\mathrm{F}_{5} \mathrm{SCF}_{2} \mathrm{CF}_{2} \mathrm{I}$ & $\begin{array}{l}\Rightarrow \mathrm{S}_{2} \mathrm{~F}_{10}+\mathrm{ICF}_{2} \mathrm{CF}_{2} \mathrm{I}+\mathrm{CF}_{2}=\mathrm{CF}_{2} /-196 \text { to } 155^{\circ} \mathrm{C} / \rightarrow \\
\quad \text { yield: } 49 \%\end{array}$ & 23 \\
\hline $\mathrm{F}_{5} \mathrm{SCF}_{2} \mathrm{CF}_{2} \mathrm{Br}$ & $\Rightarrow \mathrm{F}_{5} \mathrm{SBr}+\mathrm{CF}_{2}=\mathrm{CF}_{2} / 90^{\circ} \mathrm{C}, 2 \mathrm{~d} \rightarrow \mathrm{bp}=60-62{ }^{\circ} \mathrm{C}$ & 24 \\
\hline $\mathrm{F}_{5} \mathrm{SCF}_{2} \mathrm{CF}_{2} \mathrm{Cl}$ & $\begin{aligned} \Rightarrow & \mathrm{SF}_{5} \mathrm{Cl}+\mathrm{CF}_{2}=\mathrm{CF}_{2} /(\mathrm{PhCO})_{2} \mathrm{O}_{2}, 90^{\circ} \mathrm{C}, 10 \mathrm{~h} \rightarrow \text { yield: } \\
& 22 \% \text { to } \mathrm{C}_{2} \mathrm{~F}_{4}\end{aligned}$ & 25 \\
\hline$\left(\mathrm{CF}_{3}\right)_{3} \mathrm{GeI}$ & $\Rightarrow$ Ref. 26. Commercially available & 26 \\
\hline
\end{tabular}

Tab. 9.1-3. Perfluoroethers, perfluoroalkanones and perfluoroalkanecarboxylic acid halides

\begin{tabular}{|c|c|c|}
\hline Compound $[C, F, O(X)]$ & Remarks (" $\Rightarrow$ " for synthesis of; “•+" for reaction of; etc.) & Ref. \\
\hline 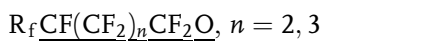 & 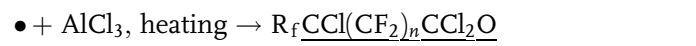 & 27 \\
\hline $\mathrm{R}_{\mathrm{f} 6} \overline{\mathrm{OR}_{\mathrm{f} 6}}$ & $\begin{array}{l}\cdot+\mathrm{AlCl}_{3} / 185^{\circ} \mathrm{C}, 14 \mathrm{~h} \rightarrow \mathrm{R}_{\mathrm{f} 5} \mathrm{COCl}(63 \%)+\mathrm{R}_{\mathrm{f} 5} \mathrm{CCl}_{3} \\
(51 \%)\end{array}$ & 28 \\
\hline $\mathrm{R}_{\mathrm{f}} \mathrm{C}(\mathrm{O}) \mathrm{CF}\left(\mathrm{CF}_{3}\right)_{2}$ & $\begin{aligned} \Rightarrow & \mathrm{R}_{\mathrm{f}} \mathrm{COF} / \mathrm{CsF} \text {-catalyst }+\mathrm{CF}_{2}=\mathrm{CFCF}_{3} \rightarrow \text { yield: } 39-75 \%, \\
& \mathrm{R}_{\mathrm{f}}=\left(\mathrm{CF}_{3}\right)_{2} \mathrm{CF} ; \mathrm{C}_{3} \mathrm{~F}_{7}, \mathrm{CF}_{3} ; \text { etc. }\end{aligned}$ & 29 \\
\hline $\mathrm{C}_{3} \mathrm{~F}_{7} \mathrm{OCF}\left(\mathrm{CF}_{3}\right) \mathrm{CF}_{2} \mathrm{OCF}\left(\mathrm{CF}_{3}\right) \mathrm{COCF}_{3}$ & $\begin{aligned} \Rightarrow & \mathrm{C}_{3} \mathrm{~F}_{7} \mathrm{OCF}\left(\mathrm{CF}_{3}\right) \mathrm{CF}_{2} \mathrm{OCF}\left(\mathrm{CF}_{3}\right) \mathrm{COF}+\mathrm{CF}_{3} \mathrm{SnMe}_{3} / \\
& 140^{\circ} \mathrm{C}, 20 \mathrm{~h} \text {, sealed tube } \rightarrow \text { yield: } 49 \%\end{aligned}$ & 30 \\
\hline $\mathrm{C}_{7} \mathrm{~F}_{15} \mathrm{COCl}$ & $\Rightarrow \mathrm{C}_{7} \mathrm{~F}_{15} \mathrm{CO}_{2} \mathrm{H}+\mathrm{SOCl}_{2} /$ DMF, heating, $4 \mathrm{~h} \rightarrow$ yield: $90 \%$ & 31 \\
\hline $\mathrm{C}_{3} \mathrm{~F}_{7} \mathrm{O}\left[\mathrm{CF}\left(\mathrm{CF}_{3}\right) \mathrm{CF}_{2} \mathrm{O}\right]_{n} \mathrm{CF}\left(\mathrm{CF}_{3}\right) \mathrm{COF}$ & $\Rightarrow \mathrm{CF}_{3} \underline{\mathrm{CFCF}_{2}} \underline{\mathrm{O}}+\mathrm{KF} /$ diglyme $\rightarrow$ acid fluorides, $n=1-4$ & 32 \\
\hline $\mathrm{C}_{7} \mathrm{~F}_{15} \mathrm{COF}$ & $\begin{array}{l}\Rightarrow \mathrm{C}_{7} \mathrm{~F}_{15} \mathrm{CO}_{2} \mathrm{H}+\mathrm{HCF}_{2} \mathrm{CF}_{2} \mathrm{~N}\left(\mathrm{CH}_{3}\right)_{2} / 0-25^{\circ} \mathrm{C}, 2 \mathrm{~h} \rightarrow \\
\quad \text { yield: } 75 \%\end{array}$ & 33 \\
\hline
\end{tabular}


Tab. 9.1-4. Perfluoroalkylsulfides and disulfides, perfluoroalkanesulfonyl halides and anhydrides

\begin{tabular}{|c|c|c|}
\hline Compound $[C, F, S(O, X)]$ & Remarks (" $\Rightarrow$ " for synthesis of; “•+" for reaction of; etc.) & Ref. \\
\hline$\left(\mathrm{C}_{3} \mathrm{~F}_{7}\right)_{2} \mathrm{~S}$ & $\Rightarrow \mathrm{C}_{3} \mathrm{~F}_{7} \mathrm{I}+\mathrm{S}_{x} / 300^{\circ} \mathrm{C}, 10 \mathrm{~h} \rightarrow$ yield: $11 \%, \mathrm{bp}=88^{\circ} \mathrm{C}$ & 34 \\
\hline$\left(\mathrm{C}_{7} \mathrm{~F}_{15}\right)_{2} \mathrm{~S}_{2}$ & $\begin{aligned} \Rightarrow & \mathrm{C}_{7} \mathrm{~F}_{15} \mathrm{I}+\mathrm{S}_{x} / 255^{\circ} \mathrm{C}, 17 \mathrm{~h} \rightarrow \text { yield: } 60 \%, \mathrm{mp}=39^{\circ} \mathrm{C}, \\
& \mathrm{bp}=247^{\circ} \mathrm{C}\end{aligned}$ & 34 \\
\hline $\mathrm{C}_{6} \mathrm{~F}_{13} \mathrm{SO}_{2} \mathrm{Cl}$ & - $+\mathrm{C}_{6} \mathrm{H}_{6} / \mathrm{RuCl}_{2}\left(\mathrm{PPh}_{3}\right)_{3} \rightarrow \mathrm{C}_{6} \mathrm{H}_{5} \mathrm{C}_{6} \mathrm{~F}_{13}$, yield: $44 \%$ & 35 \\
\hline $\mathrm{C}_{8} \mathrm{~F}_{17} \mathrm{SO}_{2} \mathrm{X}, \mathrm{X}=\mathrm{F}, \mathrm{Cl}$ & $\begin{array}{l}\Rightarrow \text { Title compounds were obtained in } \sim 98 \% \text { purity from a } \\
\text { mixture containing branched isomers by cooling to }-20{ }^{\circ} \mathrm{C} \\
\text { and filtration of the crystalline linear isomers }\end{array}$ & 36 \\
\hline $\mathrm{R}_{\mathrm{fn}} \mathrm{SO}_{2} \mathrm{Cl}$ and/or $\left(\mathrm{R}_{\mathrm{fn}} \mathrm{SO}_{2}\right)_{2} \mathrm{O}$ & $\begin{array}{l}\Rightarrow \mathrm{R}_{\mathrm{fn}} \mathrm{SO}_{3} \mathrm{~K}+\mathrm{PCl}_{5} \cdot 2 \mathrm{ZnCl}_{2} \rightarrow \text { "high yield and purity". } \\
\text { When } \mathrm{PCl}_{5} \text { reacted with perfluoroalkanesulfonic acids, } \\
\text { yields anhydrides }\end{array}$ & 37 \\
\hline$\left(c y c l o-\mathrm{C}_{2} \mathrm{~F}_{5} \mathrm{C}_{6} \mathrm{~F}_{10} \mathrm{SO}_{2}\right)_{2} \mathrm{O}$ & $\begin{array}{l}\text { - Used for the thermal perfluoroalkylation of dyes (e.g., } \\
\text { pyranthrone, } \mathrm{Cu} \text { phthalocyanine) to yield fluorous } \\
\text { derivatives for staining PTFE }\end{array}$ & 38 \\
\hline
\end{tabular}

Tab. 9.1-5. Tris (perfluoroalkyl)amines, perfluoroazomethines, perfluoroalkanecarbonitriles, tris(perfluoroalkyl)triazines and perfluoroalkyl isocyanates

\begin{tabular}{|c|c|c|}
\hline Compound $[C, F, N(O)]$ & Remarks (" $\Rightarrow$ " for synthesis of; "•+" for reaction of; etc.) & Ref. \\
\hline$\left(\mathrm{C}_{4} \mathrm{~F}_{9}\right)_{3} \mathrm{~N}$ & - An inert medium for organic reactions & 39 \\
\hline & $\begin{aligned} \Rightarrow & \text { Prepared by the defluorination of undecafluoropiperidine } \\
& \text { with steel wool at } 500{ }^{\circ} \mathrm{C} / 1 \mathrm{~atm} \text {, yield: } 40 \%\end{aligned}$ & 40 \\
\hline $\mathrm{CF}_{3} \mathrm{CF}_{2} \mathrm{CF}_{2} \mathrm{CF}_{2} \mathrm{~N}=\mathrm{CFCF}_{2} \mathrm{CF}_{2} \mathrm{CF}_{3}$ & $\begin{array}{l}\Rightarrow\left(\mathrm{C}_{4} \mathrm{~F}_{9}\right)_{3} \mathrm{~N}+\mathrm{SbF}_{5} \text { catalyst } / 120^{\circ} \mathrm{C}, 4 \mathrm{~h},\left(-\mathrm{C}_{4} \mathrm{~F}_{10}\right) \rightarrow \\
\quad \text { yield: } 67 \%\end{array}$ & 41 \\
\hline $\mathrm{C}_{7} \mathrm{~F}_{15} \mathrm{CN}$ & $\begin{aligned} \Rightarrow & \mathrm{C}_{7} \mathrm{~F}_{15} \mathrm{CONH}_{2}+\mathrm{P}_{2} \mathrm{O}_{5} / 200^{\circ} \mathrm{C} \rightarrow \text { yield: } 84 \%, \mathrm{bp}= \\
103-104^{\circ} \mathrm{C} & \end{aligned}$ & 42 \\
\hline 2,4,6-( $\left(\mathrm{C}_{7} \mathrm{~F}_{15}\right)_{3}$-1,3,5-triazine & $\Rightarrow \mathrm{C}_{7} \mathrm{~F}_{15} \mathrm{CN} /$ aniline, $120-140{ }^{\circ} \mathrm{C}, 13 \mathrm{~h} \rightarrow$ yield: $77 \%$ & 43 \\
\hline $\mathrm{R}_{\mathrm{f3}} \mathrm{NCO}$ & $\begin{aligned} \Rightarrow & \mathrm{R}_{\mathrm{f} 3} \mathrm{~N}=\mathrm{NR}_{\mathrm{f3}}+\mathrm{CO} / 300^{\circ} \mathrm{C}, 800 \mathrm{~atm}, 8 \mathrm{~h} \rightarrow \text { yield: } \\
& 11 \%+\mathrm{C}_{6} \mathrm{~F}_{14}\end{aligned}$ & 44 \\
\hline $\mathrm{R}_{\mathrm{f} 7} \mathrm{NCO}$ & $\Rightarrow \mathrm{R}_{\mathrm{f}} \mathrm{COCl}+\mathrm{Me}_{3} \mathrm{SiN}_{3} \rightarrow$ yield: $82 \%$ & 45 \\
\hline
\end{tabular}


9 Getting Started in Synthesis: A Tabular Guide to Selected Monofunctional Fluorous Compounds

Tab. 9.1-6. Perfluoroalkylmagnesium- and perfluoroalkylzinc halides; perfluoroalkyl copper(I)-, perfluoroalkyl-lithium-, and perfluoroalkyl-caesium intermediates

\begin{tabular}{|c|c|c|}
\hline Compound $[\mathrm{C}, \mathrm{F}, \mathrm{Mg} / \mathrm{Zn} / \mathrm{Cu} / \mathrm{Li} / \mathrm{Cs}]$ & Remarks (" $\Rightarrow$ " for synthesis of; "•+" for reaction of; etc.) & Ref. \\
\hline $\mathrm{R}_{\mathrm{f} 8} \mathrm{MgBr} / \mathrm{THF}$ & $\begin{aligned} \Rightarrow & \mathrm{C}_{8} \mathrm{~F}_{17} \mathrm{I}+\mathrm{PhMgBr} \text { or } \mathrm{C}_{2} \mathrm{H}_{5} \mathrm{MgBr} / \mathrm{THF} \text { at }-70^{\circ} \mathrm{C} / \rightarrow \\
& \mathrm{R}_{\mathrm{f} 8} \mathrm{MgBr} \rightarrow \mathrm{R}_{\mathrm{f} 8} \mathrm{C}\left(\mathrm{CH}_{3}\right)_{2} \mathrm{OH} \text { or } \mathrm{R}_{\mathrm{f} 8} \mathrm{C}\left(\mathrm{CF}_{3}\right)_{2} \mathrm{OH} \text {, in } 90 \\
& \text { or } 64 \% \text { yields, resp. }\end{aligned}$ & 46 \\
\hline $\mathrm{R}_{\mathrm{fn}} \mathrm{MgBr} /$ ether, $n=4,6,8,10$ & $\begin{aligned} \Rightarrow & \mathrm{C}_{n} \mathrm{~F}_{2 n+1} \mathrm{I}+\mathrm{C}_{2} \mathrm{H}_{5} \mathrm{MgBr} / \text { ether at }-35-40{ }^{\circ} \mathrm{C} / \rightarrow \\
& \mathrm{R}_{\mathrm{fn}} \mathrm{MgBr} / \text { ether; quenched by } \mathrm{PhCH}_{2} \mathrm{CH}=\mathrm{O} \text { to yield } \\
& \mathrm{PhCH}_{2} \mathrm{CH}(\mathrm{OH}) \mathrm{R}_{\mathrm{fn}} .\end{aligned}$ & 47 \\
\hline$\left(\mathrm{CF}_{3}\right)_{2} \mathrm{CF}_{2} \mathrm{ZnI} / \mathrm{THF}$ & $\bullet+\mathrm{PhCOF} / \mathrm{py} \rightarrow \operatorname{PhCOCF}\left(\mathrm{CF}_{3}\right)_{2}$, yield: $\sim 100 \%$ & 48 \\
\hline $\mathrm{R}_{\mathrm{fn}} \mathrm{Cu}$. solvent & $\begin{aligned} \Rightarrow & \mathrm{R}_{\mathrm{fn}} \mathrm{I}+2 \mathrm{Cu} / \mathrm{DMSO}, 110-120^{\circ} \mathrm{C}, 2 \mathrm{~h} \rightarrow \mathrm{R}_{\mathrm{fn}} \mathrm{Cu}, \\
& \text { yield }>80 \%\end{aligned}$ & 49 \\
\hline “ $\mathrm{C}_{8} \mathrm{~F}_{17} \mathrm{Li}$ "/ether $\left(-78^{\circ} \mathrm{C}\right)$ & $\Rightarrow$ Generated in situ: $\mathrm{C}_{8} \mathrm{~F}_{17} \mathrm{I}+\mathrm{CH}_{3} \mathrm{Li}-\mathrm{LiBr} /$ ether, $-78{ }^{\circ} \mathrm{C}$ & 50 \\
\hline $\mathrm{CF}_{3} \mathrm{CF}_{2} \mathrm{CF}_{2} \mathrm{C}\left(\mathrm{CF}_{3}\right)_{2}^{-} \mathrm{Cs}^{+}$ & $\begin{aligned} \bullet & +\mathrm{ArCH}_{2} \mathrm{Br} \rightarrow \mathrm{ArCH}_{2} \mathrm{C}\left(\mathrm{CF}_{3}\right)_{2} \mathrm{R}_{\mathrm{f} 3}, 10 \text { examples, yields: } \\
& \sim 60 \%\end{aligned}$ & 51 \\
\hline Miscellaneous F-organometallics & $\begin{aligned} \Rightarrow & \bullet+\text { Synthesis and reactivity of } F \text {-organometallics. } \\
& \text { A review }\end{aligned}$ & 52 \\
\hline
\end{tabular}

\section{Series II [hydrogen included]}

9.2.1 Hydroperfluoroalkanes, perfluoroalkylalkanes/cycloalkanes, perfluoroalkylalkenes and perfluoroalkylalkynes, perfluoroalkylarenes, (perfluoroalkyl)alkylarenes and related compounds

9.2.2 (Perfluoroalkyl)alkyl halides; perfluoroalkyl- and (perfluoroalkyl)alkyl aryl/benzyl halides

9.2.3 Perfluoroalkylalkanols and ethers

9.2.4 Fluorous mercaptanes, sulfides, sulfoxides, sulfonates, sulfonimides and selenides

9.2.5 Fluorous amines, anilines, pyridines; phosphines and phosphites

9.2.6 Fluorous boron-, silicon-, tin-, lithium-, zinc-, and magnesium compounds

9.2.7 Fluorous aldehydes and ketones

9.2.8 Fluorous carboxylic acids

9.2.9 Fluorous esters and carbonic acid derivatives

\section{Acknowledgements}

The author thanks the Hungarian Scientific Research Foundation (OTKA T 034871) and the European Contract of Research Training Network ('Fluorous Phase' HPRN-CT-2000-00002) for financial support. 
Tab. 9.2-1. Hydroperfluoroalkanes, perfluoroalkylalkanes/cycloalkanes, perfluoroalkylalkenes and perfluoroalkylalkynes, perfluoroalkylarenes, (perfluoroalkyl)alkylarenes and related compounds

\begin{tabular}{|c|c|c|}
\hline Compound $[C, F, H]$ & Remarks (" $\Rightarrow$ " for synthesis of; “•+" for reaction of; etc.) & Ref. \\
\hline $\mathrm{R}_{\mathrm{fn}} \mathrm{CF}_{2} \mathrm{H}(n=2-4)$ & $\begin{aligned} \Rightarrow & \mathrm{R}_{\mathrm{fn}} \mathrm{CF}_{2} \mathrm{I}+\mathrm{H}_{2} / \text { Raney Ni, } 60 \mathrm{~atm}, 350^{\circ} \mathrm{C} \rightarrow \text { yield: } \\
& 80 \%\end{aligned}$ & $9 \mathrm{a}$ \\
\hline$\left(\mathrm{CF}_{3}\right)_{3} \mathrm{CH}$ & $\begin{array}{l}\Rightarrow \text { Prepared as described and its thermodynamic } \\
\text { acidity studied }\end{array}$ & 53 \\
\hline $\mathrm{F}\left(\mathrm{CF}_{2}\right)_{8}\left(\mathrm{CH}_{2}\right)_{8} \mathrm{H}$ & $\begin{aligned} \Rightarrow & \mathrm{R}_{\mathrm{f} 8} \mathrm{I}+\mathrm{CH}_{2}=\mathrm{CHC}_{6} \mathrm{H}_{13} / \mathrm{AIBN} \rightarrow \mathrm{R}_{\mathrm{f} 8} \mathrm{CH}_{2} \mathrm{CH}(\mathrm{I}) \mathrm{C}_{6} \mathrm{H}_{13} ; \\
& +\mathrm{Zn} / \mathrm{HCl} \text { in } \mathrm{CH}_{3} \mathrm{OH} \rightarrow \text { title product, its gel forma- } \\
& \text { tion from alcohols studied }\end{aligned}$ & 54 \\
\hline $\mathrm{CF}_{3}-$ cyclo- $\mathrm{C}_{6} \mathrm{H}_{11}$ & $\Rightarrow \bullet+$ As a new solvent? Limits of use & 55 \\
\hline $\mathrm{R}_{\mathrm{fn}} \mathrm{CH}=\mathrm{CH}_{2}$ & $\begin{array}{l}\Rightarrow \mathrm{R}_{\mathrm{fn}} \mathrm{CH}_{2} \mathrm{CH}_{2} \mathrm{I}+\mathrm{NaOH} / \mathrm{C}_{2} \mathrm{H}_{5} \mathrm{OH}, 30^{\circ} \mathrm{C}, 1 \mathrm{~h} \rightarrow \\
\quad \text { yield: } 99 \%\end{array}$ & 56 \\
\hline $\mathrm{R}_{\mathrm{fn}} \mathrm{CH}=\mathrm{CH}_{2}, n=4,6,8,10$ & $\begin{aligned} \Rightarrow & \mathrm{R}_{\mathrm{fn}} \mathrm{I}+\mathrm{CH}_{2}=\mathrm{CHSi}\left(\mathrm{CH}_{3}\right)_{3} \rightarrow \mathrm{R}_{\mathrm{fn}} \mathrm{CH}_{2} \mathrm{CHISi}\left(\mathrm{CH}_{3}\right)_{3} ; \\
& +\mathrm{Bu}_{4} \mathrm{NF} \rightarrow\end{aligned}$ & 57 \\
\hline $\mathrm{R}_{\mathrm{f} 8} \mathrm{CH}_{2} \mathrm{CH}=\mathrm{CH}_{2}$ & $\Rightarrow \mathrm{R}_{\mathrm{f} 8} \mathrm{I}+\mathrm{CH}_{2}=\mathrm{CHCH}_{2} \mathrm{Cl} / \mathrm{AIBN} \rightarrow$ title olefin + adduct & 58 \\
\hline$\left(\mathrm{CF}_{3}\right)_{3} \mathrm{CCH}_{2} \mathrm{CH}=\mathrm{CH}_{2}$ & $\begin{array}{l}\Rightarrow\left(\mathrm{CF}_{3}\right)_{2} \mathrm{C}=\mathrm{CF}_{2} / \mathrm{CsF}+\mathrm{CH}_{2}=\mathrm{CHCH}_{2} \mathrm{I} / \text { diglyme } \rightarrow \\
\quad \text { yield: } 80 \%\end{array}$ & 59 \\
\hline $\mathrm{CF}_{3}\left(\mathrm{CF}_{2}\right)_{2} \mathrm{C}\left(\mathrm{CF}_{3}\right)_{2} \mathrm{CH}_{2} \mathrm{CH}=\mathrm{CH}_{2}$ & $\begin{array}{l}\Rightarrow \text { Alkylation of perfluoro-2-methyl-2-pentylcarbanion with } \\
\text { alkyl and allyl halides. Study the effect of solvents and } \\
\mathrm{F}^{-} \text {-sources }\end{array}$ & 60 \\
\hline$\left(\mathrm{C}_{2} \mathrm{~F}_{5}\right)_{2} \mathrm{C}\left(\mathrm{CF}_{3}\right) \mathrm{OCH}_{2} \mathrm{CH}=\mathrm{CH}_{2}$ & $\begin{aligned} \Rightarrow & \left(\mathrm{C}_{2} \mathrm{~F}_{5}\right)_{2} \mathrm{C}\left(\mathrm{CF}_{3}\right) \mathrm{OK}+\mathrm{CH}_{2}=\mathrm{CHCH}_{2} \mathrm{Br} / \mathrm{CH}_{3} \mathrm{CN} \text {, reflux } \rightarrow \\
& \text { bp } 120^{\circ} \mathrm{C}\end{aligned}$ & 61 \\
\hline$\left(\mathrm{CF}_{3}\right)_{2} \mathrm{NCH}_{2} \mathrm{CH}=\mathrm{CH}_{2}$ & $\begin{aligned} \Rightarrow & \left(\mathrm{CF}_{3}\right)_{2} \mathrm{~N}^{-} \mathrm{Cs}^{+}+\mathrm{CH}_{2}=\mathrm{CHCH}_{2} \mathrm{Br} \rightarrow \text { yield: } 44 \%, \mathrm{bp}= \\
& 42{ }^{\circ} \mathrm{C}\end{aligned}$ & 62 \\
\hline$n-\mathrm{C}_{4} \mathrm{~F}_{9} \mathrm{CH}=\mathrm{CHC}_{4} \mathrm{~F}_{9-n}$ & $\begin{array}{l}\text { - + Remains unaffected when heated at } 130^{\circ} \mathrm{C} \text { with } \\
\mathrm{Et}_{2} \mathrm{NH} \text { or } \mathrm{Br}_{2} \text { for several weeks, by } m \text {-chloroperbenzoic } \\
\text { acid }\end{array}$ & 63 \\
\hline $\mathrm{R}_{\mathrm{fn}} \mathrm{C} \equiv \mathrm{CH}, n=4,6$ & $\begin{aligned} \Rightarrow & \mathrm{R}_{\mathrm{fn}} \mathrm{I}+\mathrm{HC} \equiv \mathrm{CC}\left(\mathrm{CH}_{3}\right)_{2} \mathrm{OH} / \text { electrocatal. } \rightarrow \\
& \mathrm{R}_{\mathrm{fn}} \mathrm{CH}=\mathrm{CIC}\left(\mathrm{CH}_{3}\right)_{2} \mathrm{OH} \rightarrow \mathrm{R}_{\mathrm{fn}} \mathrm{C} \equiv \mathrm{CC}\left(\mathrm{CH}_{3}\right)_{2} \mathrm{OH} \rightarrow \\
& \text { overall yield: } 55-60 \%\end{aligned}$ & 64 \\
\hline $\mathrm{C}_{8} \mathrm{~F}_{17} \mathrm{C}_{6} \mathrm{H}_{5}$, etc. & $\begin{array}{l}\Rightarrow \mathrm{C}_{8} \mathrm{~F}_{17} \mathrm{I}+\mathrm{C}_{6} \mathrm{H}_{6} / \mathrm{K}_{2} \mathrm{CO}_{3}, 2 \% \mathrm{Ru}-\mathrm{C}, 30 \mathrm{~h}, 170{ }^{\circ} \mathrm{C} \rightarrow \\
\quad \text { yield: } 89 \%\end{array}$ & 65 \\
\hline $\mathrm{R}_{\mathrm{f} 8} \mathrm{Ph}$ & $\begin{aligned} \Rightarrow & \mathrm{PhH}+\mathrm{R}_{\mathrm{f} 8} \mathrm{~N}=\mathrm{NR}_{\mathrm{f} 8} / \mathrm{CF}_{2} \mathrm{ClCFCl}_{2}, h v, 2 \mathrm{~h} \rightarrow \text { yield: } \\
& 70 \%\end{aligned}$ & 66 \\
\hline \multirow[t]{2}{*}{$\mathrm{R}_{\mathrm{fn}} \mathrm{R},(\mathrm{R}=\mathrm{Ar}$, Het $)$} & $\begin{array}{l}\Rightarrow 2 \mathrm{R}_{\mathrm{fn}} \mathrm{I}+\mathrm{ArH} / \sim 250{ }^{\circ} \mathrm{C}, 15 \mathrm{~h} \rightarrow \mathrm{R}_{\mathrm{fn}} \mathrm{Ar}+\mathrm{R}_{\mathrm{fn}} \mathrm{H}+\mathrm{I}_{2}, \\
\quad \text { yield: } 60-65 \%\end{array}$ & 67 \\
\hline & $\begin{array}{l}\Rightarrow \mathrm{R}_{\mathrm{fn}} \mathrm{I}+\mathrm{ArH}+{ }^{\mathrm{t}} \mathrm{Bu}_{2} \mathrm{O}_{2}, \sim 150{ }^{\circ} \mathrm{C}, 8 \mathrm{~h} \rightarrow \mathrm{R}_{\mathrm{fn}} \mathrm{Ar} \\
\quad \text { yield: } 40-95 \%\end{array}$ & $67 d$ \\
\hline $\begin{array}{l}\mathrm{R}_{\mathrm{f} 8} \mathrm{C}_{6} \mathrm{H}_{4} \mathrm{CF}_{3} ; \mathrm{R}_{\mathrm{f} 8} \mathrm{C}_{6} \mathrm{H}_{3}\left(\mathrm{CF}_{3}\right)_{2} \\
\quad(o-, m \text { - and } p-; \text { and } 1,3,5 \text {-isomers })\end{array}$ & $\begin{aligned} \Rightarrow & \mathrm{R}_{\mathrm{fn}} \mathrm{I}+\mathrm{ArI} / \mathrm{Cu}, \mathrm{DMSO} / 130-135{ }^{\circ} \mathrm{C}, 6 \mathrm{~h} \rightarrow \text { yield: } \\
& 48-84 \%\end{aligned}$ & 68 \\
\hline $\mathrm{F}_{5} \mathrm{~S}\left(\mathrm{CF}_{2}\right)_{6} \mathrm{C}_{6} \mathrm{H}_{5}$ & $\begin{aligned} \Rightarrow & \mathrm{F}_{5} \mathrm{~S}\left(\mathrm{CF}_{2}\right)_{6} \mathrm{I}+\mathrm{C}_{6} \mathrm{H}_{6}\left(160-165^{\circ} \mathrm{C} / 14 \mathrm{~d}\right) \rightarrow \text { yield: } \\
& 51 \% \text { by GC }\end{aligned}$ & 69 \\
\hline $\mathrm{R}_{\mathrm{f} 8} \mathrm{CH}_{2} \mathrm{CH}_{2} \mathrm{Ph}$ & $\begin{aligned} \Rightarrow & \mathrm{PhMgCl}+\mathrm{R}_{\mathrm{f} 8} \mathrm{CH}_{2} \mathrm{CH}_{2} \mathrm{I} / \mathrm{CuBr}-\mathrm{THF}, 18 \text { h, r.t. } \rightarrow \\
& \text { yield: } 89 \%\end{aligned}$ & 70 \\
\hline $\mathrm{R}_{\mathrm{f} 10} \mathrm{CH}_{2} \mathrm{CH}_{2} \mathrm{Ph}$ & $\begin{aligned} \Rightarrow & \mathrm{PhB}(\mathrm{OH})_{2}+\mathrm{R}_{\mathrm{f} 10} \mathrm{CH}_{2} \mathrm{CH}_{2} \mathrm{I} / \mathrm{Pd}\left(\mathrm{PPh}_{3}\right)_{4}, \mathrm{NaHCO}_{3}, \\
& \mathrm{H}_{2} \mathrm{O}-\mathrm{CH}_{3} \mathrm{OCH}_{2} \mathrm{CH}_{2} \mathrm{OCH}_{3}, 5 \mathrm{~h} \rightarrow \text { yield: } 89 \%\end{aligned}$ & 71 \\
\hline
\end{tabular}


9 Getting Started in Synthesis: A Tabular Guide to Selected Monofunctional Fluorous Compounds

Tab. 9.2-1 (continued)

\begin{tabular}{|c|c|c|}
\hline Compound $[C, F, H]$ & Remarks (“" $\Rightarrow$ " for synthesis of; “•+" for reaction of; etc.) & Ref. \\
\hline $\mathrm{R}_{\mathrm{fn}} \mathrm{Ar}$ & $\Rightarrow \mathrm{ArBr}+\mathrm{R}_{\mathrm{fn}} \mathrm{I} / \mathrm{Cu}, \mathrm{DMSO} \rightarrow$ good yields & 72 \\
\hline $\mathrm{R}_{\mathrm{fn}} \mathrm{CH}_{2} \mathrm{CH}_{2} \mathrm{Ar}$ via $\mathrm{R}_{\mathrm{fn}} \mathrm{CH}=\mathrm{CHAr}$ & $\begin{aligned} \Rightarrow & \mathrm{ArX}+\mathrm{CH}_{2}=\mathrm{CHR}_{\mathrm{fn}} / \text { Pd-cat } \rightarrow \mathrm{ArCH}=\mathrm{CHR}_{\mathrm{fn}} ; \\
& +\mathrm{H}_{2} / \mathrm{Pd}-\mathrm{C} \rightarrow\end{aligned}$ & 73 \\
\hline $\begin{array}{r}\left(\mathrm{R}_{\mathrm{fn}} \mathrm{CH}_{2} \mathrm{CH}=\mathrm{CH}\right)_{x} \mathrm{Ar}, n=6,8,10 \\
\left(\mathrm{R}_{\mathrm{f} n} \mathrm{CH}_{2} \mathrm{CH}_{2} \mathrm{CH}_{2}\right)_{x} \mathrm{Ar} ; x=1-3\end{array}$ & $\begin{aligned} \Rightarrow & {\left[\mathrm{R}_{\mathrm{fn}} \mathrm{CH}_{2} \mathrm{CH}_{2} \mathrm{PPh}_{3}\right]^{+} \mathrm{I}^{-}+\mathrm{Ar}(\mathrm{CHO})_{x} \rightarrow } \\
& \left(\mathrm{R}_{\mathrm{fn}} \mathrm{CH}_{2} \mathrm{CH}=\mathrm{CH}\right)_{x} \mathrm{Ar} ;+\mathrm{H}_{2} / \text { cat } \rightarrow \text { overall yield: } \\
& \quad 68-90 \%\end{aligned}$ & 74 \\
\hline
\end{tabular}

Tab. 9.2-2. (Perfluoroalkyl)alkyl halides; perfluoroalkyl- and (perfluoroalkyl)alkyl aryl/benzyl halides

\begin{tabular}{|c|c|c|}
\hline Compound $[C, F, H, X]$ & Remarks (" $\Rightarrow$ " for synthesis of; "•+" for reaction of; etc.) & Ref. \\
\hline $\mathrm{R}_{\mathrm{f} 3} \mathrm{CH}_{2} \mathrm{I}$ & $\begin{array}{l}\Rightarrow \mathrm{R}_{\mathrm{f} 3} \mathrm{CH}_{2} \mathrm{OTs}+\mathrm{KI} / \text { diethylene glycol, } T>190{ }^{\circ} \mathrm{C} \rightarrow \\
\quad \text { yield: } 84 \%\end{array}$ & 75 \\
\hline $\mathrm{R}_{\mathrm{fn}} \mathrm{CH}_{2} \mathrm{CH}_{2} \mathrm{I}$ & $\begin{array}{l}\Rightarrow \mathrm{R}_{\mathrm{fn}} \mathrm{I}+\mathrm{CH}_{2}=\mathrm{CH}_{2} / 290-390{ }^{\circ} \mathrm{C} \text { for } n=4,6,8,10 \rightarrow \\
\quad \text { yield: } \sim 95 \%\end{array}$ & 76,77 \\
\hline $\mathrm{R}_{\mathrm{f} 6} \mathrm{CH}_{2} \mathrm{CH}_{2} \mathrm{CH}_{2} \mathrm{I}$ & $\begin{aligned} \Rightarrow & \mathrm{R}_{\mathrm{f} 6}\left(\mathrm{CH}_{2}\right)_{3} \mathrm{OH}+\mathrm{KI}, \mathrm{P}_{2} \mathrm{O}_{5} / \mathrm{H}_{3} \mathrm{PO}_{4} \rightarrow \text { yield: } 88 \% \\
& (72 \% \text { conversion })\end{aligned}$ & 56 \\
\hline $\mathrm{R}_{\mathrm{f} 8} \mathrm{CH}_{2} \mathrm{CH}_{2} \mathrm{CH}_{2} \mathrm{I}$ & $\begin{aligned} \Rightarrow & \mathrm{R}_{\mathrm{f} 8}\left(\mathrm{CH}_{2}\right)_{3} \mathrm{OH}+\mathrm{KI}, \mathrm{P}_{2} \mathrm{O}_{5} / \mathrm{H}_{3} \mathrm{PO}_{4} ; \mathrm{P}_{2} \mathrm{O}_{5} / \mathrm{SiO}_{2} \rightarrow \text { yield: } \\
& 70-85 \%\end{aligned}$ & 78 \\
\hline $\mathrm{R}_{\mathrm{f} 8} \mathrm{CH}_{2} \mathrm{CH}_{2} \mathrm{CH}_{2} \mathrm{CH}_{2} \mathrm{I}$ & $\begin{array}{l}\Rightarrow \mathrm{R}_{\mathrm{f} 8}\left(\mathrm{CH}_{2}\right)_{3} \mathrm{OH}+\mathrm{KI}, \mathrm{P}_{2} \mathrm{O}_{5} / \mathrm{H}_{3} \mathrm{PO}_{4}\left(120^{\circ} \mathrm{C} / 4 \mathrm{~h}\right) \rightarrow \\
\text { yield: } 92 \%\end{array}$ & 79 \\
\hline $\mathrm{F}_{5} \mathrm{~S}\left(\mathrm{CF}_{2} \mathrm{CF}_{2}\right)_{3} \mathrm{CH}_{2} \mathrm{CH}_{2} \mathrm{I}$ & $\Rightarrow \mathrm{CH}_{2}=\mathrm{CH}_{2}+\mathrm{F}_{5} \mathrm{~S}\left(\mathrm{CF}_{2} \mathrm{CF}_{2}\right)_{3} \mathrm{I} /{ }^{\mathrm{t}} \mathrm{Bu}_{2} \mathrm{O}_{2} \rightarrow$ yield: $28 \%$ & 80 \\
\hline $1,3,5-\mathrm{BrC}_{6} \mathrm{H}_{3}\left(\mathrm{R}_{\mathrm{f} 8}\right)_{2}$ & $\begin{array}{l}\Rightarrow \text { 1,3,5- } \mathrm{Br}_{3} \mathrm{C}_{6} \mathrm{H}_{3}+2 \mathrm{R}_{\mathrm{f} 8} \mathrm{I}+4 \mathrm{Cu} / \mathrm{DMF}, 120^{\circ} \mathrm{C}, 18 \mathrm{~h} \rightarrow \\
\quad \text { yield: } 60 \%\end{array}$ & 81 \\
\hline $1,3,5-\mathrm{BrC}_{6} \mathrm{H}_{3}\left(\mathrm{R}_{\mathrm{f} 10}\right)_{2}$ & $\begin{array}{l}\Rightarrow m-\left(\mathrm{R}_{\mathrm{f} 10}\right)_{2} \mathrm{C}_{6} \mathrm{H}_{4}+\mathrm{NBS} / \mathrm{H}_{2} \mathrm{SO}_{4}-\mathrm{CF}_{3} \mathrm{CO}_{2} \mathrm{H}, 50^{\circ} \mathrm{C}, 8 \mathrm{~h} \rightarrow \\
\quad \text { yield: } 94 \%\end{array}$ & 82 \\
\hline $\begin{array}{l}1,2,4,6-\mathrm{IC}_{6} \mathrm{H}_{2}\left[\left(\mathrm{CH}_{2}\right)_{3} \mathrm{R}_{\mathrm{f} 8}\right]_{3} \\
\quad 1,2,4-, 1,2,5-; 1,3,4- \\
\mathrm{IC}_{6} \mathrm{H}_{3}\left[\left(\mathrm{CH}_{2}\right)_{3} \mathrm{R}_{\mathrm{f} 8}\right]_{2}\end{array}$ & $\begin{aligned} \Rightarrow & \mathrm{C}_{6} \mathrm{H}_{3}\left(\mathrm{CH}_{2} \mathrm{CH}_{2} \mathrm{CH}_{2} \mathrm{R}_{\mathrm{f} 8}\right)_{3} \text { or } \mathrm{C}_{6} \mathrm{H}_{4}\left(\mathrm{CH}_{2} \mathrm{CH}_{2} \mathrm{CH}_{2} \mathrm{R}_{\mathrm{f} 8}\right)_{2}+ \\
& \mathrm{I}_{2} / \mathrm{H}_{5} \mathrm{IO}_{6} \text { in } \mathrm{AcOH}, \mathrm{H}_{2} \mathrm{SO}_{4} / \mathrm{H}_{2} \mathrm{O} \rightarrow \text { yields of respective } \\
& \text { iodoarenes: } 61-97 \%\end{aligned}$ & 83 \\
\hline $3,5-\left(\mathrm{R}_{\mathrm{f} 8}\right)_{2} \mathrm{C}_{6} \mathrm{H}_{3} \mathrm{CH}_{2} \mathrm{Br}$ & $\Rightarrow 3,5-\left(\mathrm{R}_{\mathrm{f} 8}\right)_{2} \mathrm{C}_{6} \mathrm{H}_{3} \mathrm{CH}_{2} \mathrm{OH}+\mathrm{PBr}_{3} / \mathrm{THF} \rightarrow$ yield: $62 \%$ & 84 \\
\hline
\end{tabular}


Tab. 9.2-3. Perfluoroalkylalkanols and ethers

\begin{tabular}{|c|c|c|}
\hline Compound $[C, F, H, O]$ & $\begin{array}{l}\text { Remarks (" } \Rightarrow \text { " for synthesis of; "• } \bullet " \text { "for } \\
\text { reaction of; etc.) }\end{array}$ & Ref. \\
\hline $\mathrm{C}_{3} \mathrm{~F}_{7} \mathrm{OCF}\left(\mathrm{CF}_{3}\right) \mathrm{CF}_{2} \mathrm{OCF}\left(\mathrm{CF}_{3}\right) \mathrm{CH}_{2} \mathrm{OH}$ & $\begin{array}{l}\Rightarrow \mathrm{C}_{3} \mathrm{~F}_{7} \mathrm{OCF}\left(\mathrm{CF}_{3}\right) \mathrm{CF}_{2} \mathrm{OCF}\left(\mathrm{CF}_{3}\right) \mathrm{CO}_{2} \mathrm{CH}_{3}+\mathrm{NaBH}_{4} / \\
\quad \text { ether } \rightarrow(81 \%)\end{array}$ & 85 \\
\hline$\left(\mathrm{CF}_{3}\right)_{3} \mathrm{CCH}_{2} \mathrm{OH}$ & $\begin{array}{l}\Rightarrow\left(\mathrm{CF}_{3}\right)_{3} \mathrm{CH}+\mathrm{CH}_{2} \mathrm{O} / \mathrm{Et}_{3} \mathrm{~N}, 100^{\circ} \mathrm{C}, 55 \mathrm{~h} \rightarrow \mathrm{no} \\
\quad \text { yield data given }\end{array}$ & 86 \\
\hline $\mathrm{R}_{\mathrm{fn}} \mathrm{CH}_{2} \mathrm{CH}_{2} \mathrm{CH}_{2} \mathrm{OH}, n=6,8,10$ & $\begin{array}{l}\Rightarrow \text { One pot synthesis from } \mathrm{R}_{\mathrm{fn}} \mathrm{I} \text { and triallyl borate, } \\
\text { yield: } 74-79 \%\end{array}$ & 87 \\
\hline$\left(\mathrm{C}_{6} \mathrm{~F}_{13} \mathrm{CH}_{2} \mathrm{CH}_{2}\right)_{2} \mathrm{C}\left(\mathrm{CH}_{2} \mathrm{OH}\right)_{2}$ & $\Rightarrow$ Preparation of and use for acetalyzation & 88 \\
\hline$\left(\mathrm{R}_{\mathrm{f} 8} \mathrm{CH}_{2} \mathrm{CH}_{2}\right)_{2} \mathrm{CHOH}$ & $\begin{array}{l}\Rightarrow \mathrm{C}_{8} \mathrm{~F}_{17} \mathrm{CH}_{2} \mathrm{CH}_{2} \mathrm{I}+\mathrm{Mg} / \text { ultrasound; }+\mathrm{HCO}_{2} \mathrm{Et} \rightarrow \\
\text { yield: } 93 \%\end{array}$ & 89 \\
\hline $\begin{array}{l}\mathrm{R}_{\mathrm{f} 8} \mathrm{CH}_{2} \mathrm{CH}_{2} \mathrm{C}\left(\mathrm{CH}_{3}\right)_{2} \mathrm{OH} \\
\quad\left[\mathrm{R}_{\mathrm{f} 6}\left(\mathrm{CH}_{2}\right)_{n}\right]_{2} \mathrm{C}\left(\mathrm{CH}_{3}\right) \mathrm{OH}, n=2,3\end{array}$ & $\begin{aligned} \Rightarrow & \mathrm{R}_{\mathrm{f}}\left(\mathrm{CH}_{2}\right)_{n} \mathrm{MgX}+\mathrm{Me}_{2} \mathrm{CO} \text { or EtOAc } \rightarrow \text { used for the } \\
& \text { synthesis of }{ }^{\mathrm{F}} \text { Boc-transfer reagents }\end{aligned}$ & 90 \\
\hline $\begin{array}{l}\mathrm{R}_{\mathrm{f} 6} \mathrm{CH}_{2} \mathrm{CH}_{2} \mathrm{C}\left(\mathrm{CH}_{3}\right)_{2} \mathrm{OH} \\
\quad\left[\mathrm{R}_{\mathrm{fn}}\left(\mathrm{CH}_{2}\right)_{2}\right]_{2} \mathrm{C}\left(\mathrm{CH}_{3}\right) \mathrm{OH}, n=6,8\end{array}$ & $\begin{array}{l}\Rightarrow \text { Conveniently prepared by the reaction of } \\
\text { appropriate Grignard reagents with acetone and } \\
\text { ethyl acetate, respectively }\end{array}$ & 91 \\
\hline $\mathrm{R}_{6} \mathrm{CH}_{2} \mathrm{CH}_{2} \mathrm{C}\left(\mathrm{CH}_{3}\right)_{2} \mathrm{OH}$ & $\begin{array}{l}\Rightarrow \mathrm{R}_{\mathrm{f} 6} \mathrm{I}+\mathrm{CH}_{2}=\mathrm{CHC}\left(\mathrm{CH}_{3}\right)_{2} \mathrm{OH} / \mathrm{Cu}, 120^{\circ} \mathrm{C} \rightarrow \\
\quad \mathrm{R}_{\mathrm{f} 6} \mathrm{CH}_{2} \mathrm{CH}(\mathrm{I}) \mathrm{C}\left(\mathrm{CH}_{3}\right)_{2} \mathrm{OH}(83 \%) ;+\mathrm{Bu}_{3} \mathrm{SnH} \rightarrow \\
\quad \text { yield: } 67 \%\end{array}$ & 92 \\
\hline $\mathrm{R}_{\mathrm{fn}} \mathrm{CH}_{2} \mathrm{CH}_{2} \mathrm{C}\left(\mathrm{CH}_{3}\right)_{2} \mathrm{OH}, n=4,6,8$ & $\begin{aligned} \Rightarrow & \mathrm{R}_{\mathrm{fn}} \mathrm{CH}=\mathrm{CH}_{2}+\left(\mathrm{CH}_{3}\right)_{2} \mathrm{CHOH} / \text { acetone, } h v, \text { r.t., } \\
& 3 \mathrm{~d} \rightarrow \text { yield: } 91 \%\end{aligned}$ & 93 \\
\hline$\left(\mathrm{R}_{\mathrm{f} 6} \mathrm{CH}_{2} \mathrm{CH}_{2}\right)_{3} \mathrm{COH}$ & $\begin{array}{l}\Rightarrow \mathrm{C}_{6} \mathrm{~F}_{17} \mathrm{CH}_{2} \mathrm{CH}_{2} \mathrm{I}+\mathrm{Mg} / \text { ether; }+\mathrm{CH}_{3} \mathrm{OC}(\mathrm{O}) \mathrm{OCH}_{3} \rightarrow \\
\quad \text { yield: } 40 \%\end{array}$ & 94 \\
\hline $3,5-\left(\mathrm{C}_{8} \mathrm{~F}_{17}\right)_{2} \mathrm{C}_{6} \mathrm{H}_{3} \mathrm{CH}_{2} \mathrm{OH}$ & $\begin{aligned} \Rightarrow & 1,3-\left(\mathrm{R}_{\mathrm{f} 8}\right)_{2} \mathrm{C}_{6} \mathrm{H}_{3} \mathrm{CO}_{2} \mathrm{CH}_{3}+\mathrm{LiAlH}_{4} / \mathrm{Et}_{2} \mathrm{O} \\
& 5 \mathrm{~h} \text { reflux } \rightarrow \text { yield: } 90 \%\end{aligned}$ & 68 \\
\hline $\mathrm{C}_{8} \mathrm{~F}_{17} \frac{\mathrm{Si}\left(\mathrm{CH}_{3}\right)_{3}}{\mathrm{CH}_{3}}$ & $\begin{array}{l}\Rightarrow \mathrm{CH}_{3} \mathrm{COSi}\left(\mathrm{CH}_{3}\right)_{3}+\mathrm{C}_{8} \mathrm{~F}_{17} \mathrm{I} / \mathrm{C}_{2} \mathrm{H}_{5} \mathrm{MgBr},-45^{\circ} \mathrm{C} \text { to } \\
\quad \text { r.t. } \rightarrow \text { yield: } 45 \%, \mathrm{mp}=29^{\circ} \mathrm{C}\end{array}$ & 95 \\
\hline $\mathrm{R}_{\mathrm{f} 6} \mathrm{CH}_{2} \mathrm{CH}_{2} \mathrm{OCH}\left(\mathrm{CH}_{3}\right) \mathrm{CH}_{2} \mathrm{CH}\left(\mathrm{CH}_{3}\right)_{2}$ & $\begin{array}{l}\Rightarrow \bullet+\text { A new fluorous/organic amphiphilic ether } \\
\text { solvent }\end{array}$ & 96 \\
\hline$\left(\mathrm{CF}_{3}\right)_{3} \mathrm{CCH}_{2} \mathrm{OCH}_{2} \mathrm{C}\left(\mathrm{CF}_{3}\right)_{3}$ & $\begin{aligned} \Rightarrow & \left(\mathrm{CF}_{3}\right)_{2} \mathrm{C}=\mathrm{CF}_{2} / \mathrm{CsF}+\left(\mathrm{ClCH}_{2}\right)_{2} \mathrm{O} \rightarrow \text { yield: } 71 \% \\
& \mathrm{bp}=156-157^{\circ} \mathrm{C}\end{aligned}$ & 97 \\
\hline$\left(\mathrm{CF}_{3}\right)_{3} \mathrm{CCH}_{2} \mathrm{OCH}_{3}$ & $\begin{array}{l}\Rightarrow\left(\mathrm{CF}_{3}\right)_{2} \mathrm{C}=\mathrm{CF}_{2}+\mathrm{ClCH}_{2} \mathrm{OCH}_{3} / \mathrm{CsF} \text {, diglym, } \\
\quad 70^{\circ} \mathrm{C} \rightarrow \text { yield: } 90 \% \\
\bullet \\
\quad+\mathrm{SO}_{3} / \mathrm{H}_{2} \mathrm{SO}_{4} \text {; r.t., } 1 \mathrm{~h} \rightarrow\left(\mathrm{CF}_{3}\right)_{3} \mathrm{CCH}_{2} \mathrm{OH} \text {, yield: } \\
\quad 45 \%\end{array}$ & 98 \\
\hline$\left(\mathrm{CF}_{3}\right)_{3} \mathrm{COCH}_{3},\left(\mathrm{CF}_{3}\right)_{3} \mathrm{COC}_{2} \mathrm{H}_{5}$ & $\Rightarrow\left(\mathrm{CF}_{3}\right)_{3} \mathrm{CONa}+\mathrm{R}_{2} \mathrm{SO}_{4} /$ tetraglyme $\rightarrow$ yield: $\sim 95 \%$ & 99 \\
\hline $\mathrm{R}_{\mathrm{fn}_{n}} \mathrm{CH}_{2} \mathrm{CH}(-\mathrm{O}-) \mathrm{CH}_{2}, n=4,6,8$ & $\begin{aligned} \Rightarrow & \left(\text { a) } \mathrm{R}_{\mathrm{fn}} \mathrm{I}+\mathrm{CH}_{2}=\mathrm{CHCH}_{2} \mathrm{OAc} \rightarrow\right. \\
& \mathrm{R}_{\mathrm{fn}} \mathrm{CH}_{2} \mathrm{CH}(\mathrm{I}) \mathrm{CH}_{2} \mathrm{OAc}+\mathrm{R}_{\mathrm{fn}} \mathrm{CH}_{2} \mathrm{CH}(\mathrm{OAc}) \mathrm{CH}_{2} \mathrm{I} ; \\
& (\mathrm{b})+\mathrm{KOH} / \text { hexane } \rightarrow \text { yield: } 94-96 \%\end{aligned}$ & 100 \\
\hline
\end{tabular}


9 Getting Started in Synthesis: A Tabular Guide to Selected Monofunctional Fluorous Compounds

Tab. 9.2-4. Fluorous mercaptanes, sulfides, sulfoxides, sulfonates, sulfonimides and selenides

\begin{tabular}{|c|c|c|}
\hline Compound $[\mathrm{C}, \mathrm{F}, \mathrm{H}, \mathrm{S} / \mathrm{Se}(\mathrm{O})]$ & Remarks (“" $\Rightarrow$ " for synthesis of; “•+" for reaction of; etc.) & Ref. \\
\hline $\mathrm{R}_{\mathrm{fn}} \mathrm{CH}_{2} \mathrm{SH}, n=2,3$ & $\begin{array}{l}\Rightarrow \mathrm{R}_{\mathrm{fn}} \mathrm{COX}(\mathrm{X}=\mathrm{H}, \mathrm{Cl})+\mathrm{H}_{2} \mathrm{~S}, 200^{\circ} \mathrm{C}, 3000 \mathrm{~atm} \rightarrow \\
\quad \text { yield: } 39-54 \%\end{array}$ & 101 \\
\hline $\mathrm{R}_{\mathrm{f} 6} \mathrm{CH}_{2} \mathrm{CH}_{2} \mathrm{SH}$ & $\begin{array}{l}\Rightarrow \mathrm{R}_{\mathrm{f} 6}\left(\mathrm{CH}_{2}\right)_{2} \mathrm{I}+\mathrm{H}_{2} \mathrm{NCSNH}_{2} \text { dioxane } \rightarrow \text { yield: } 94 \%, \\
\text { unlike alcohol type solvents no foul-smelling } \\
\text { byproducts are formed in dioxane! }\end{array}$ & 102 \\
\hline $\mathrm{R}_{\mathrm{f} 8} \mathrm{CH}_{2} \mathrm{CH}_{2} \mathrm{CH}_{2} \mathrm{SH}$ & $\begin{array}{l}\text { - }+\left(\mathrm{C}_{8} \mathrm{H}_{17}\right)_{4} \mathrm{NAuCl}_{4} / \mathrm{CF}_{3} \mathrm{Ph}+\mathrm{NaBH}_{4} / \mathrm{H}_{2} \mathrm{O} \rightarrow \\
\left(\mathrm{Au}_{x}\right)\left[\mathrm{HS}\left(\mathrm{CH}_{2}\right)_{3} \mathrm{R}_{\mathrm{f} 8}\right]_{Y} \\
\text { First example for an isolable and fluorous soluble } \\
\quad \text { gold nanocluster }\end{array}$ & 103 \\
\hline $\mathrm{F}_{5} \mathrm{~S}\left(\mathrm{CF}_{2}\right)_{6} \mathrm{CH}_{2} \mathrm{CH}_{2} \mathrm{SH}$ & $\begin{array}{l}\Rightarrow \mathrm{F}_{5} \mathrm{~S}\left(\mathrm{CF}_{2}\right)_{6}\left(\mathrm{CH}_{2}\right)_{2} \mathrm{I}+\mathrm{NaH} / \mathrm{AcSH}-\mathrm{THF} \rightarrow \\
\quad \mathrm{F}_{5} \mathrm{~S}\left(\mathrm{CF}_{2}\right)_{6}\left(\mathrm{CH}_{2}\right)_{2} \mathrm{SAc} ;+\mathrm{LiAlH}_{4} / \mathrm{THF} \rightarrow \text { overall } \\
\quad \text { yield: } 90 \%\end{array}$ & 104 \\
\hline $\mathrm{R}_{\mathrm{f} 7} \mathrm{SCH}_{3}$ & $\Rightarrow \mathrm{R}_{\mathrm{f} 7} \mathrm{C}(\mathrm{O}) \mathrm{SCH}_{3} / \mathrm{CH}_{3} \mathrm{CN}, h v /(-\mathrm{CO}) \rightarrow$ yield: $81 \%$ & 105 \\
\hline $\mathrm{R}_{\mathrm{f} 8} \mathrm{CH}_{2} \mathrm{CH}_{2} \mathrm{SCH}_{3}$ & $\begin{aligned} \Rightarrow & \mathrm{R}_{\mathrm{f} 8} \mathrm{CH}_{2} \mathrm{CH}_{2} \mathrm{SCOCH}_{3}+\mathrm{NaOCH}_{3} ; \mathrm{CH}_{3} \mathrm{I} \rightarrow \text { yield: }\end{aligned}$ & 106 \\
\hline $\mathrm{R}_{\mathrm{fn}} \mathrm{SCH}_{2} \mathrm{CH}_{2} \mathrm{OH} ; n=4,6$ & $\begin{array}{l}\Rightarrow \mathrm{HO}\left(\mathrm{CH}_{2}\right)_{2} \mathrm{SH}+\mathrm{R}_{\mathrm{fn}} \mathrm{I} / \mathrm{Na}_{2} \mathrm{SO}_{3}, \mathrm{HCO}_{2} \mathrm{Na}, \mathrm{DMF}-\mathrm{H}_{2} \mathrm{O} \text {, } \\
\quad \text { r.t. } \rightarrow\end{array}$ & 107 \\
\hline$\left[\mathrm{R}_{\mathrm{f} 8}\left(\mathrm{CH}_{2}\right)_{n}\right]_{2} \mathrm{~S}, n=2,3$ & $\Rightarrow \mathrm{R}_{\mathrm{f} 8}\left(\mathrm{CH}_{2}\right)_{n} \mathrm{I}+\mathrm{Li}_{2} \mathrm{~S} / \mathrm{THF} \rightarrow$ yields: $67-71 \%$ & 108 \\
\hline $\operatorname{ArSR}_{\mathrm{fn}}, n=3$ & $\Rightarrow \mathrm{ArSH}+\mathrm{R}_{\mathrm{fn}} \mathrm{I}+\mathrm{NaOH}, \mathrm{PhCH}_{2} \mathrm{NEt}_{3} \mathrm{Cl} \rightarrow$ yield: $83 \%$ & 109 \\
\hline $\begin{array}{l}\operatorname{ArSR}_{\mathrm{fn}}, \text { e.g.: } \mathrm{Ar}=p-\mathrm{CH}_{3} \mathrm{C}_{6} \mathrm{H}_{4} \\
\quad n=6\end{array}$ & $\begin{array}{l}\Rightarrow \text { (a) } \mathrm{ArSH}+\mathrm{N}_{\left(\mathrm{C}_{2} \mathrm{H}_{5}\right)_{3}+\mathrm{R}_{\mathrm{f} 6} \mathrm{Br} / \mathrm{DMF}, h v \rightarrow \text { yield: }} \\
\quad 52 \% \\
\Rightarrow \text { (b) } \mathrm{ArSK}+\mathrm{R}_{\mathrm{f} 6} \mathrm{Br} / \mathrm{DMF} \sim 3 \mathrm{~atm} \rightarrow \text { yield: } 77 \%\end{array}$ & 110,111 \\
\hline $\begin{array}{l}\mathrm{R}_{\mathrm{f} 8}\left(\mathrm{CH}_{2}\right)_{n} \mathrm{SO}\left(\mathrm{CH}_{2}\right)_{n} \mathrm{R}_{\mathrm{f} 8} \\
\quad n=2,3\end{array}$ & $\Rightarrow\left[\mathrm{R}_{\mathrm{f} 8}\left(\mathrm{CH}_{2}\right)_{n}\right]_{2} \mathrm{~S}+\mathrm{CH}_{3} \mathrm{CO}_{3} \mathrm{H} \rightarrow$ yield: $80-85 \%$ & 108 \\
\hline $\begin{array}{l}\mathrm{R}_{\mathrm{fn}} \mathrm{CH}_{2} \mathrm{CH}_{2} \mathrm{~S}(\mathrm{O}) \mathrm{CH}_{3} \\
\quad n=4,6\end{array}$ & $\begin{aligned} \Rightarrow & \left(\mathrm{CH}_{3}\right)_{2} \mathrm{~S}_{2} / \mathrm{NaBH}_{4}+\mathrm{R}_{\mathrm{fn}}\left(\mathrm{CH}_{2}\right)_{2} \mathrm{I} \rightarrow \mathrm{R}_{\mathrm{fn}}\left(\mathrm{CH}_{2}\right)_{2} \mathrm{SCH}_{3} ; \\
& +\mathrm{H}_{2} \mathrm{O}_{2} / \mathrm{CH}_{3} \mathrm{OH} \rightarrow \text { overall yield: } 71 \%\end{aligned}$ & 112 \\
\hline $\mathrm{R}_{\mathrm{f} 7} \mathrm{CH}_{2} \mathrm{OSO}_{2} \mathrm{CF}_{3}$ & $\Rightarrow \mathrm{R}_{\mathrm{f} 7} \mathrm{CH}_{2} \mathrm{OH}+\left(\mathrm{CF}_{3} \mathrm{SO}_{2}\right)_{2} \mathrm{O} /$ py, $\mathrm{CH}_{2} \mathrm{Cl}_{2} \rightarrow$ yield: $92 \%$ & 113 \\
\hline $\mathrm{R}_{\mathrm{f} 7} \mathrm{CH}_{2} \mathrm{OSO}_{2} \mathrm{C}_{4} \mathrm{~F}_{9}$ & $\Rightarrow \mathrm{R}_{\mathrm{f} 7} \mathrm{CH}_{2} \mathrm{OH}+\mathrm{R}_{\mathrm{f} 4} \mathrm{SO}_{2} \mathrm{~F} / \mathrm{TEA}$, ether $\rightarrow$ yield: $95 \%$ & 114 \\
\hline $\mathrm{R}_{\mathrm{f} 8} \mathrm{CH}_{2} \mathrm{CH}_{2} \mathrm{CH}_{2} \mathrm{OTs}$ & $\begin{array}{l}\Rightarrow \mathrm{R}_{\mathrm{f} 8} \mathrm{CH}_{2} \mathrm{CH}_{2} \mathrm{CH}_{2} \mathrm{OH}+\mathrm{TsCl} / \mathrm{CH}_{2} \mathrm{Cl}_{2} \text {-aq. } \mathrm{NaOH} \rightarrow \\
\quad \text { yield: } 70 \%\end{array}$ & 115 \\
\hline$\left(\mathrm{R}_{\mathrm{fn}} \mathrm{SO}_{2}\right)_{2} \mathrm{NH}, n=2,4,8$ & $\begin{array}{l}\Rightarrow \text { Products were obtained after high-vacuum } \\
\text { sublimation or short-path distillation from conc. } \\
\mathrm{H}_{2} \mathrm{SO}_{4}\end{array}$ & 116 \\
\hline 2,4-( $\left(\mathrm{R}_{\mathrm{f} 8}\right)_{2} \mathrm{C}_{6} \mathrm{H}_{3} \mathrm{SeC}_{4} \mathrm{H}_{9}$ & $\begin{aligned} \Rightarrow & \text { 2,4- } \mathrm{I}_{2} \mathrm{C}_{6} \mathrm{H}_{3} \mathrm{NH}_{2}+\mathrm{R}_{\mathrm{f} 8} \mathrm{I} / \mathrm{Cu}, \mathrm{DMSO}, 120{ }^{\circ} \mathrm{C} \rightarrow \\
& \text { 2,4-( }\left(\mathrm{R}_{\mathrm{f} 8}\right)_{2} \mathrm{C}_{6} \mathrm{H}_{3} \mathrm{NH}_{2} ;+\mathrm{NaNO}_{2}, \mathrm{HBr}, \mathrm{CuBr} \rightarrow \\
& \text { 2,4-( }\left(\mathrm{R}_{\mathrm{f} 8}\right)_{2} \mathrm{C}_{6} \mathrm{H}_{3} \mathrm{Br} ;+\mathrm{C}_{4} \mathrm{H}_{9} \mathrm{SeLi} / \mathrm{THF},-80 \text { to } \\
& -25{ }^{\circ} \mathrm{C} \rightarrow 2,4-\left(\mathrm{R}_{\mathrm{f} 8}\right)_{2} \mathrm{C}_{6} \mathrm{H}_{3} \mathrm{SeC}_{4} \mathrm{H}_{9} \text {, overall yield: } 36 \%\end{aligned}$ & 117 \\
\hline 3,5-( $\left(\mathrm{R}_{\mathrm{f} 8}\right)_{2} \mathrm{C}_{6} \mathrm{H}_{3} \mathrm{SeC}_{4} \mathrm{H}_{9}$ & $\begin{array}{l}\Rightarrow \text { Prepared by multiple step synthesis from } \\
\text { 3,5- } \mathrm{I}_{2} \mathrm{C}_{6} \mathrm{H}_{3} \mathrm{NH}_{2} \text { and used as a catalyst in Bayer-Villiger } \\
\text { oxidation }\end{array}$ & 118 \\
\hline
\end{tabular}


Tab. 9.2-5. Fluorous amines, anilines, pyridines; phosphines and phosphites

\begin{tabular}{|c|c|c|}
\hline Compound $[C, F, H, N / P(O)]$ & $\begin{array}{l}\text { Remarks (" } \Rightarrow \text { " for synthesis of; " } \bullet+" \text { for } \\
\text { reaction of; etc.) }\end{array}$ & Ref. \\
\hline $\mathrm{R}_{\mathrm{f} 7} \mathrm{CH}_{2} \mathrm{NH}_{2},\left(\mathrm{R}_{\mathrm{f}} \mathrm{CH}_{2}\right)_{2} \mathrm{NH}$ & $\begin{array}{l}\Rightarrow \text { Precursors of tetrasubstituted ureas, thermally } \\
\text { stable fluids, recoverable in } 99 \% \text { after a } 63 \mathrm{~h} \text { at } \\
270{ }^{\circ} \mathrm{C} \text { test }\end{array}$ & 119 \\
\hline$\left(\mathrm{R}_{\mathrm{f} 9} \mathrm{CH}_{2}\right)_{2} \mathrm{NH}$ & $\begin{aligned} \Rightarrow & \left(\mathrm{R}_{\mathrm{f} 9} \mathrm{CO}\right)_{2} \mathrm{NH}+\mathrm{LiAlH}_{4} \rightarrow \text { no yield data reported, } \\
& \mathrm{mp}=85-86^{\circ} \mathrm{C}\end{aligned}$ & 120 \\
\hline$\left[\left(\mathrm{CF}_{3}\right)_{3} \mathrm{C}\right]_{2} \mathrm{NH}$ & $\Rightarrow$ multistep synthesis $/ \mathrm{bp}=100-101^{\circ} \mathrm{C} /$ & 121 \\
\hline $\mathrm{R}_{\mathrm{f} 7} \mathrm{CH}_{2} \mathrm{~N}\left(\mathrm{CH}_{3}\right)_{2}$ & $\begin{array}{l}\Rightarrow \mathrm{R}_{\mathrm{f} 7} \mathrm{CSN}\left(\mathrm{CH}_{3}\right)_{2} ;+\mathrm{BH}_{3} / \text { diglyme } \rightarrow \text { yield: } 59 \%, \\
\quad \mathrm{GC} \text { purity: } 99 \%\end{array}$ & 122 \\
\hline$\left(\mathrm{CF}_{2}\right)_{4} \mathrm{NCH}_{2} \mathrm{CF}_{2} \mathrm{CF}_{3}$ & $\Rightarrow \frac{\mathrm{CF}_{2}}{\text { yield: }} \frac{\mathrm{CF}_{2}}{71 \%} \frac{\mathrm{CF}_{2}}{\mathrm{CF}=\mathrm{N}}+\mathrm{KF}+\mathrm{CF}_{3} \mathrm{CF}_{2} \mathrm{CH}_{2} \mathrm{OSO}_{2} \mathrm{CF}_{3} \rightarrow$ & 123 \\
\hline $\mathrm{CF}_{3} \mathrm{CF}_{2}\left(\mathrm{CF}_{3}\right) \mathrm{NCH}_{3}$ & $\begin{array}{l}\Rightarrow \mathrm{CF}_{3} \mathrm{CF}=\mathrm{NCF}_{3}+\mathrm{CsF}+\left(\mathrm{CH}_{3}\right)_{2} \mathrm{SO}_{4} / \mathrm{CH}_{3} \mathrm{CN} \rightarrow \\
\quad \text { yield: } 46 \%\end{array}$ & 124 \\
\hline$\left[\left(\mathrm{CF}_{3}\right)_{3} \mathrm{CCH}_{2}\right]_{3} \mathrm{~N}$ & $\begin{array}{l}\Rightarrow\left(\mathrm{CF}_{3}\right)_{2} \mathrm{C}=\mathrm{CF}_{2}+\mathrm{CsF}+\left(\mathrm{ClCH}_{2}\right)_{3} \mathrm{~N} / \text { diglyme } \rightarrow \\
\quad \text { yield: } 32 \%\end{array}$ & 97 \\
\hline $\mathrm{R}_{\mathrm{fn}} \mathrm{CH}_{2} \mathrm{CH}_{2} \mathrm{NH}_{2}, n=2,4,6,8$ & $\Rightarrow \mathrm{R}_{\mathrm{fn}} \mathrm{CH}_{2} \mathrm{CH}_{2} \mathrm{I}+\mathrm{NaN}_{3} \rightarrow \mathrm{R}_{\mathrm{fn}} \mathrm{CH}_{2} \mathrm{CH}_{2} \mathrm{~N}_{3} ; \mathrm{H}_{2} / \mathrm{Pt} \rightarrow$ & 125 \\
\hline $\begin{array}{l}\mathrm{NH}_{3-x}\left[\left(\mathrm{CH}_{2}\right)_{m} \mathrm{R}_{\mathrm{f} 8}\right]_{x}, m=3-5 \\
\quad x=1,2 \mathrm{~N}\left[\left(\mathrm{CH}_{2}\right)_{m} \mathrm{R}_{\mathrm{f} 8}\right]_{3}=\mathrm{N}\left(\mathrm{R}_{\mathrm{fh}}\right)_{3}\end{array}$ & $\begin{aligned} \Rightarrow & \mathrm{R}_{\mathrm{f} 8}\left(\mathrm{CH}_{2}\right)_{m-1} \mathrm{CHO}+\mathrm{NH}\left(\mathrm{R}_{\mathrm{fh}}\right)_{2} \text { or } \mathrm{H}_{2} \mathrm{NCH}_{2} \mathrm{Ph} / \\
& \mathrm{Na}(\mathrm{AcO})_{3} \mathrm{BH} \rightarrow \mathrm{N}\left(\mathrm{R}_{\mathrm{fh}}\right)_{3} \text { or } \mathrm{NH}_{2-x}\left(\mathrm{CH}_{2} \mathrm{Ph}\right) \\
& {\left[\left(\mathrm{CH}_{2}\right)_{m} \mathrm{R}_{\mathrm{f} 8}\right]_{x} ;+\mathrm{H}_{2} / \mathrm{Pd}-\mathrm{C} \rightarrow 78-91 \% }\end{aligned}$ & 126 \\
\hline$\left[\left(\mathrm{R}_{\mathrm{f} 6} \mathrm{CH}_{2} \mathrm{CH}_{2}\right)_{3} \mathrm{SiCH}_{2} \mathrm{CH}_{2} \mathrm{CH}_{2}\right]_{2} \mathrm{NH}$ & $\begin{array}{l}\Rightarrow\left[\left(\mathrm{R}_{\mathrm{f} 6}\left(\mathrm{CH}_{2}\right)_{2} \mathrm{Si}\left(\mathrm{CH}_{2}\right)_{3}\right]_{2} \mathrm{NCOCF}_{3}+\mathrm{LiAlH}_{4} / \text { ether } \rightarrow\right. \\
\quad \text { yield: } 97 \% \\
\text { • }+ \text { RNCO } \rightarrow \text { fluorous soluble ureas, easy to remove } \\
\quad \text { by extraction }\end{array}$ & 127 \\
\hline$\left(\mathrm{R}_{8} \mathrm{CH}_{2} \mathrm{CH}_{2} \mathrm{CH}_{2}\right)_{2} \mathrm{NCH}_{3}$ & $\begin{array}{l}\Rightarrow \mathrm{R}_{\mathrm{f} 8}\left(\mathrm{CH}_{2}\right)_{3} \mathrm{NCH}_{3}+\mathrm{R}_{\mathrm{f} 8}\left(\mathrm{CH}_{2}\right)_{3} \mathrm{I} / \mathrm{THF}, 60^{\circ} \mathrm{C}, 24 \mathrm{~h} \rightarrow \\
\quad \text { yield: } 84 \%\end{array}$ & 128 \\
\hline $\begin{array}{l}{\left[\left(\mathrm{R}_{\mathrm{f} 8} \mathrm{CH}_{2} \mathrm{CH}_{2} \mathrm{CH}_{2}\right)_{3} \mathrm{NCH}_{3}\right]^{+} \mathrm{CH}_{3} \mathrm{OSO}_{3}^{-}=} \\
\quad \mathrm{R}_{\mathrm{F}}^{+} \mathrm{CH}_{3} \mathrm{OSO}_{3}^{-}\end{array}$ & $\begin{aligned} \Rightarrow & \left.\bullet+\mathrm{Na}_{12}\left\{\left[\mathrm{WZnM}_{2}\left(\mathrm{H}_{2} \mathrm{O}\right)_{2}\right]\left(\mathrm{ZnW}_{9} \mathrm{O}_{34}\right)_{2}\right]\right\} \rightarrow \text { fluorous } \\
& \text { soluble salts of polyoxometalates: e.g. } \\
& \left.\left(\mathrm{R}_{\mathrm{F}}^{+}\right)_{12}\left[\mathrm{WZn}_{3}\left(\mathrm{H}_{2} \mathrm{O}\right)_{2}\right]\left(\mathrm{ZnW}_{9} \mathrm{O}_{34}\right)_{2}\right]^{12-}\end{aligned}$ & 129 \\
\hline $\begin{array}{l}\text { 2,4,6-( }\left(\mathrm{R}_{\mathrm{f} 8}\right)_{3} \mathrm{C}_{6} \mathrm{H}_{2} \mathrm{NH}_{2} \\
\left.\text { 4,2,6-( } \mathrm{CF}_{3}\right)\left(\mathrm{R}_{\mathrm{f} 8}\right)_{2} \mathrm{C}_{6} \mathrm{H}_{2} \mathrm{NH}_{2} \\
4,2,6-\left(\mathrm{Me}_{3} \mathrm{C}\right)\left(\mathrm{R}_{\mathrm{f} 8}\right)_{2} \mathrm{C}_{6} \mathrm{H}_{2} \mathrm{NH}_{2}\end{array}$ & $\begin{array}{l}\Rightarrow \mathrm{C}_{6} \mathrm{H}_{5} \mathrm{~N}\left(\mathrm{CH}_{3}\right)_{2}, 4-\mathrm{CF}_{3} \mathrm{C}_{6} \mathrm{H}_{4} \mathrm{NH}_{2} \text { or } \mathrm{Me}_{3} \mathrm{CC}_{6} \mathrm{H}_{4} \mathrm{NH}_{2}+ \\
\quad \mathrm{R}_{\mathrm{f} 8} \mathrm{I} / \mathrm{Cu}_{2} \mathrm{O} \text {, DMSO, } 130{ }^{\circ} \mathrm{C}, 1 \text { to } 3 \mathrm{~d} \rightarrow \text { yield: } 45,65 \\
\quad \text { and } 27 \%\end{array}$ & 130 \\
\hline $\begin{array}{l}\text { 2,6- and 3,5- }\left(\mathrm{C}_{8} \mathrm{~F}_{17} \mathrm{CH}_{2} \mathrm{CH}_{2}\right)_{2} \mathrm{C}_{5} \mathrm{H}_{3} \mathrm{~N} \\
s-\left[\mathrm{R}_{\mathrm{f} 8}\left(\mathrm{CH}_{2}\right)_{2}\right]_{3} \mathrm{py}, 2,6-\left[\mathrm{R}_{\mathrm{f} 8}\left(\mathrm{CH}_{2}\right)_{3}\right]_{2} \mathrm{py}\end{array}$ & $\begin{array}{l}\Rightarrow \mathrm{C}_{8} \mathrm{~F}_{17} \mathrm{CH}_{2} \mathrm{CH}_{2} \mathrm{ZnI} / \mathrm{THF}+\mathrm{Br}_{2} \mathrm{py} / \mathrm{Cl}_{2} \mathrm{Pd}\left(\mathrm{PPh}_{3}\right)_{3} \rightarrow \\
\quad \text { yield: } 31-85 \% \\
\Rightarrow \text { py }(\mathrm{CHO})_{x} \rightarrow\left(\mathrm{R}_{\mathrm{f} 8} \mathrm{CH}_{2} \mathrm{CH}=\mathrm{CH}\right)_{x} \text { py; }+\mathrm{H}_{2} / \mathrm{Pd}-\mathrm{C} \rightarrow \\
\quad \text { yield: } 93 \%\end{array}$ & 131 \\
\hline $2,6-\left(\mathrm{R}_{\mathrm{f} 6}\right)_{2} \mathrm{py}$ & $\Rightarrow 2,6-\mathrm{Br}_{2} \mathrm{py}+\mathrm{C}_{6} \mathrm{~F}_{13} \mathrm{I} / \mathrm{Cu}$, DMSO, $125^{\circ} \mathrm{C} \rightarrow$ yield: $89 \%$ & 132 \\
\hline $\begin{array}{l}\left.\mathrm{P}\left[\left(\mathrm{CH}_{2}\right)_{Y} \mathrm{R}_{\mathrm{fn}}\right)_{3}\right]_{3}, y=2, n=6,8,10 \\
\quad y=3,4, n=8\end{array}$ & $\begin{array}{l}\Rightarrow \mathrm{PH}_{3}+\mathrm{CH}_{2}=\mathrm{CH}\left(\mathrm{CH}_{2}\right)_{x} \mathrm{R}_{\mathrm{fn}} / \mathrm{AIBN} \text { or VAZO } \rightarrow \\
\quad \text { yield: } 63-81 \%\end{array}$ & 133,134 \\
\hline $\mathrm{R}_{\mathrm{f} 8}\left(\mathrm{CH}_{2}\right)_{m} \mathrm{PH}_{2} ; \mathrm{R}_{\mathrm{f} 8}\left(\mathrm{CH}_{2}\right)_{m} \mathrm{P}\left[\left(\mathrm{CH}_{2}\right)_{m^{\prime}} \mathrm{R}_{\mathrm{f} 8}\right]_{2}$ & $\begin{aligned} \Rightarrow & \mathrm{LiPH}_{2}+\mathrm{R}_{\mathrm{f} 8}\left(\mathrm{CH}_{2}\right)_{m} \mathrm{I} \rightarrow \mathrm{R}_{\mathrm{f} 8}\left(\mathrm{CH}_{2}\right)_{m} \mathrm{PH}_{2} ; \\
& +\mathrm{R}_{\mathrm{f} 8}\left(\mathrm{CH}_{2}\right)_{m^{\prime}-2} \mathrm{CH}=\mathrm{CH}_{2} / \mathrm{AIBN} \text { or VAZO } \rightarrow \operatorname{good} \\
& \text { yields: } m=2-4, m / m^{\prime}=3 / 2,2 / 3,4 / 3,3 / 4\end{aligned}$ & 79 \\
\hline$\left(\mathrm{R}_{\mathrm{f} 8} \mathrm{CH}_{2} \mathrm{CH}_{2} \mathrm{O}\right)_{3} \mathrm{P}$ & $\Rightarrow \mathrm{R}_{\mathrm{f} 8} \mathrm{CH}_{2} \mathrm{CH}_{2} \mathrm{OH}+\mathrm{PCl}_{3} /$ py-ether $\rightarrow$ yield: $55 \%$ & 133 \\
\hline
\end{tabular}


Tab. 9.2-6. Fluorous boron-, silicon-, tin-, lithium-, zinc-, and magnesium compounds

\begin{tabular}{|c|c|c|}
\hline Compound $[C, F, H, B / S i / S n / M e t a l]$ & Remarks (" $\Rightarrow$ "for synthesis of; “•+" for reaction of; etc.) & Ref. \\
\hline$\left(\mathrm{R}_{\mathrm{f} 10}\right)_{2} \mathrm{C}_{6} \mathrm{H}_{3} \mathrm{~B}(\mathrm{OH})_{2}$ & $\begin{aligned} \Rightarrow & \text { Multistep synthesis: } 1,3-\mathrm{C}_{6} \mathrm{H}_{4} \mathrm{I}_{2} \rightarrow 1,3-\mathrm{C}_{6} \mathrm{H}_{4}\left(\mathrm{R}_{\mathrm{f} 10}\right)_{2} \rightarrow \\
& 1,3,5-\mathrm{BrC}_{6} \mathrm{H}_{3}\left(\mathrm{R}_{\mathrm{f} 10}\right)_{2} \rightarrow 1,3,5-(\mathrm{RO})_{2} \mathrm{BC}_{6} \mathrm{H}_{3}\left(\mathrm{R}_{\mathrm{f} 10}\right)_{2} \rightarrow\end{aligned}$ & 82 \\
\hline $\mathrm{NaB}\left[\mathrm{C}_{6} \mathrm{H}_{4}\left(\mathrm{SiMe}_{2} \mathrm{CH}_{2} \mathrm{CH}_{2} \mathrm{C}_{6} \mathrm{~F}_{13}\right)-p\right]_{4}$ & $\Rightarrow \mathrm{ArBr}+{ }^{\mathrm{t}} \mathrm{BuLi} /$ ether, $-78^{\circ} \mathrm{C} \rightarrow$ ArLi/ether; & 135 \\
\hline $\mathrm{NaB}\left[\mathrm{C}_{6} \mathrm{H}_{4} \mathrm{C}_{6} \mathrm{~F}_{13}-p\right]_{4}$ & $\begin{array}{l}+\mathrm{BF}_{3} \cdot \mathrm{O}\left(\mathrm{C}_{2} \mathrm{H}_{5}\right)_{2} ; \rightarrow \mathrm{LiB}[\mathrm{Ar}]_{4} / \text { ether; }+\mathrm{NaCl}-\mathrm{H}_{2} \mathrm{O} \rightarrow \\
\text { overall yield: } 70-80 \%\end{array}$ & 136 \\
\hline $\mathrm{NaB}\left[\mathrm{C}_{6} \mathrm{H}_{3}\left(\mathrm{C}_{6} \mathrm{~F}_{13}\right)_{2}-3,5\right]_{4}$ & $\begin{aligned} \Rightarrow & \text { Multistep procedure starting from } 1,3-\mathrm{C}_{6} \mathrm{H}_{4} \mathrm{I}_{2} \text { and } \\
& \mathrm{C}_{6} \mathrm{~F}_{13} \mathrm{I}\end{aligned}$ & 137 \\
\hline$\left(\mathrm{R}_{\mathrm{f} 6} \mathrm{CH}_{2} \mathrm{CH}_{2}\right)_{3} \mathrm{SiH}$ & $\begin{array}{l}\cdot+\left(\mathrm{CH}_{2}=\mathrm{CHCH}_{2}\right)_{2} \mathrm{NCOCF}_{3} / \mathrm{H}_{2} \mathrm{PtCl}_{6}, 80^{\circ} \mathrm{C}, 12 \mathrm{~h} \rightarrow \\
\quad\left[\left(\mathrm{R}_{\mathrm{f} 6} \mathrm{CH}_{2} \mathrm{CH}_{2}\right)_{3} \mathrm{SiCH}_{2} \mathrm{CH}_{2} \mathrm{CH}_{2}\right]_{2} \mathrm{NCOCF}_{3} \text {, yield: } \\
\quad 33-37 \%\end{array}$ & 127 \\
\hline \multirow[t]{2}{*}{$\mathrm{R}_{\mathrm{fn}} \mathrm{Si}\left(\mathrm{CH}_{3}\right)_{3}, n=1-3$} & $\Rightarrow\left(\mathrm{CH}_{3}\right)_{3} \mathrm{SiCl}+\mathrm{R}_{\mathrm{fn}} \mathrm{Br} / \mathrm{PhCN} ;+\left[\left(\mathrm{C}_{2} \mathrm{H}_{5}\right)_{2} \mathrm{~N}\right]_{3} \mathrm{P} / \mathrm{PhCN} \rightarrow$ & 138 \\
\hline & $\begin{array}{l}\text { - + Useful perfluoroalkyl-anion transfer reagents } \\
\Rightarrow\left(\mathrm{R}_{f 6} \mathrm{CH}_{2} \mathrm{CH}_{2}\right)_{3} \mathrm{SiBr}+\mathrm{CH}_{2} \mathrm{CH}=\mathrm{CH}_{2} \mathrm{MgBr} \rightarrow \text { yield: }\end{array}$ & $\begin{array}{l}139 \\
140\end{array}$ \\
\hline 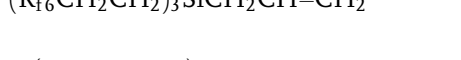 & $\begin{array}{l}\Rightarrow\left(\mathrm{K}_{\mathrm{f} 6} \mathrm{CH}_{2} \mathrm{CH}_{2}\right)_{3} \mathrm{SiBr}+\mathrm{CH}_{2} \mathrm{CH}=\mathrm{CH}_{2} \mathrm{MgBr} \rightarrow \text { yield. } \\
\quad 98 \%\end{array}$ & \\
\hline$p-\left(\mathrm{R}_{\mathrm{f} 6} \mathrm{CH}_{2} \mathrm{CH}_{2}\right)_{3} \mathrm{SiC}_{6} \mathrm{H}_{4} \mathrm{CH}_{2} \mathrm{Br}$ & $\begin{aligned} \Rightarrow & {\left[\mathrm{R}_{\mathrm{f} 6}\left(\mathrm{CH}_{2}\right)_{2}\right]_{3} \mathrm{SiBr}+p \text {-TolMgBr } \rightarrow\left[\mathrm{R}_{\mathrm{f} 6}\left(\mathrm{CH}_{2}\right)_{2}\right]_{3} \text { SiTol- } p } \\
& +\mathrm{Br}_{2} \rightarrow\end{aligned}$ & 141 \\
\hline$\left(\mathrm{R}_{\mathrm{f} 10} \mathrm{CH}_{2} \mathrm{CH}_{2}\right)_{3} \mathrm{SiC}_{6} \mathrm{H}_{4} \mathrm{CO}_{2} \mathrm{H}$ & $\Rightarrow$ Multistep synthesis from $\mathrm{R}_{\mathrm{f} 10} \mathrm{CH}_{2} \mathrm{CH}_{2} \mathrm{I}$ and $\mathrm{HSiCl}_{3}$ & 142 \\
\hline $\mathrm{R}_{\mathrm{fn}}\left(\mathrm{CH}_{2}\right)_{2}(i-\mathrm{Pr})_{2} \mathrm{SiBr}, n=6,8$ & $\begin{aligned} \Rightarrow & \mathrm{R}_{\mathrm{fn}}\left(\mathrm{CH}_{2}\right)_{2} \mathrm{Si}\left({ }^{\mathrm{i}} \mathrm{Pr}\right)_{2} \mathrm{H}+\mathrm{Br}_{2} / \mathrm{FC}-72 \rightarrow \bullet \text { Used up } \\
& \text { immediately }\end{aligned}$ & 143 \\
\hline $\begin{array}{l}\left(\mathrm{R}_{\mathrm{fn}} \mathrm{CH}_{2} \mathrm{CH}_{2}\right)_{3-x} \mathrm{Si}\left(\mathrm{CH}_{3}\right)_{x} \mathrm{Br} \\
\quad n=6,8 ; x=0,1,2\end{array}$ & $\begin{aligned} \Rightarrow & \mathrm{R}_{\mathrm{fn}}\left(\mathrm{CH}_{2}\right)_{2} \mathrm{I}+\mathrm{Mg} / \text { ether } \rightarrow \mathrm{R}_{\mathrm{fn}}\left(\mathrm{CH}_{2}\right)_{2} \mathrm{MgI} \\
& +\mathrm{HSi}\left(\mathrm{CH}_{3}\right)_{2-x} \mathrm{Cl}_{x+1} \rightarrow\left(\mathrm{R}_{\mathrm{fn}} \mathrm{CH}_{2} \mathrm{CH}_{2}\right)_{3-x} \mathrm{Si}\left(\mathrm{CH}_{3}\right)_{x} \mathrm{H} \\
& +\mathrm{Br}_{2} / \text { hexane } \rightarrow \text { product }\end{aligned}$ & 144 \\
\hline $\mathrm{R}_{\mathrm{f} 10} \mathrm{CH}_{2} \mathrm{CH}_{2} \mathrm{Si}\left(\mathrm{CH}_{3}\right)_{2} \mathrm{Cl}$ & $\begin{array}{l}\Rightarrow \mathrm{R}_{\mathrm{f} 10} \mathrm{CH}=\mathrm{CH}_{2}+\mathrm{HSi}\left(\mathrm{CH}_{3}\right)_{2} \mathrm{Cl} / \mathrm{RhCl}\left(\mathrm{PPh}_{3}\right)_{3} \rightarrow \\
\quad \text { yield: } 85 \%\end{array}$ & 145 \\
\hline $\mathrm{R}_{\mathrm{f} 3} \mathrm{C}\left(\mathrm{CF}_{3}\right)_{2} \mathrm{CH}_{2} \mathrm{CH}_{2} \mathrm{CH}_{2} \mathrm{SiMe}_{2} \mathrm{Cl}$ & $\begin{aligned} \Rightarrow & \mathrm{R}_{\mathrm{f} 3} \mathrm{C}\left(\mathrm{CF}_{3}\right)_{2} \mathrm{CH}_{2} \mathrm{CH}=\mathrm{CH}_{2}+\mathrm{HSiMe}_{2} \mathrm{Cl} / \mathrm{H}_{2} \mathrm{PtCl}_{6} / \\
& \text { heating } \rightarrow 81 \%\end{aligned}$ & 146 \\
\hline$\left[\mathrm{R}_{\mathrm{f} 8} \mathrm{CH}_{2} \mathrm{CH}_{2}\left(\mathrm{CH}_{3}\right)_{2} \mathrm{Si}\right]_{2} \mathrm{O}$ & $\begin{aligned} \Rightarrow & \mathrm{R}_{\mathrm{f} 8} \mathrm{I}+\left[\mathrm{CH}_{2}=\mathrm{CH}\left(\mathrm{CH}_{3}\right)_{2} \mathrm{Si}\right]_{2} \mathrm{O} / \mathrm{AIBN} \rightarrow \\
& {\left[\mathrm{R}_{\mathrm{f} 8} \mathrm{CH}_{2} \mathrm{CHI}\left(\mathrm{CH}_{3}\right)_{2} \mathrm{Si}\right]_{2} \mathrm{O} ;+\mathrm{Bu}_{3} \mathrm{SnH}, 80^{\circ} \mathrm{C}, 2 \mathrm{~h} \rightarrow }\end{aligned}$ & 147 \\
\hline $\begin{array}{l}\mathrm{SiO}_{2}(-\mathrm{O})_{3} \mathrm{Si}\left(\mathrm{CH}_{2}\right)_{3} \mathrm{NHC}(\mathrm{O})- \\
\quad \mathrm{CF}\left(\mathrm{CF}_{3}\right) \mathrm{OCF}\left(\mathrm{CF}_{3}\right) \mathrm{OCF}\left(\mathrm{CF}_{3}\right) \mathrm{OC}_{3} \mathrm{~F}_{7}\end{array}$ & $\Rightarrow$ Silica-(-O $)_{3} \mathrm{Si}\left(\mathrm{CH}_{2}\right)_{3} \mathrm{NH}_{2}+\mathrm{R}_{\mathrm{f}} \mathrm{OR}_{\mathrm{f}} \mathrm{C}(\mathrm{O}) \mathrm{F} \rightarrow$ & 148 \\
\hline$\left[\mathrm{R}_{\mathrm{f} 6}\left(\mathrm{CH}_{2}\right)_{3}\right]_{3} \mathrm{SnCH}_{2} \mathrm{CH}=\mathrm{CH}_{2}$ & - + Useful fluorous allyl-transfer reagent & 149 \\
\hline $4-\mathrm{RC}_{6} \mathrm{H}_{4} \mathrm{Sn}\left[\left(\mathrm{CH}_{2}\right)_{2} \mathrm{R}_{\mathrm{f} 6}\right]_{3}$ & $\begin{aligned} \Rightarrow & \bullet+\mathrm{ArOSO}_{2} \mathrm{CF}_{3} / \mathrm{DMF} \mathrm{THF}, \mathrm{PdCl}_{2}\left(\mathrm{PPh}_{3}\right)_{2}, \mathrm{LiCl} \rightarrow \\
& 4-\mathrm{RC}_{6} \mathrm{H}_{4} \mathrm{Ar}\end{aligned}$ & 150 \\
\hline$\left(\mathrm{R}_{\mathrm{f} 6} \mathrm{CH}_{2} \mathrm{CH}_{2}\right)_{3} \mathrm{SnR}, \mathrm{R}=\mathrm{Ph}, \mathrm{Br}, \mathrm{H}$ & $\begin{array}{l}\Rightarrow \mathrm{Cl}_{3} \mathrm{SnPh}+\mathrm{R}_{\mathrm{f} 6} \mathrm{CH}_{2} \mathrm{CH}_{2} \mathrm{MgI} \rightarrow\left(\mathrm{R}_{\mathrm{f} 6} \mathrm{CH}_{2} \mathrm{CH}_{2}\right)_{3} \mathrm{SnPh} ; \\
\quad+\mathrm{Br}_{2} \rightarrow\left(\mathrm{R}_{\mathrm{f} 6} \mathrm{CH}_{2} \mathrm{CH}_{2}\right)_{3} \mathrm{SnBr} ;+\mathrm{LiAlH}_{4} \rightarrow \text { overall } \\
\quad \text { yield: } 65 \%\end{array}$ & 151 \\
\hline$\left(\mathrm{R}_{\mathrm{f} 6} \mathrm{CH}_{2} \mathrm{CH}_{2}\right)_{3} \mathrm{SnN}_{3}$ & $\begin{array}{l}\Rightarrow\left(\mathrm{R}_{\mathrm{f} 6} \mathrm{CH}_{2} \mathrm{CH}_{2}\right)_{3} \mathrm{SnBr}+\mathrm{NaN}_{3}, \text { ether- } \mathrm{H}_{2} \mathrm{O}, 25^{\circ} \mathrm{C} \rightarrow \\
\quad \text { yield: } 97 \%\end{array}$ & 152 \\
\hline $\mathrm{Sn}\left[\mathrm{N}\left(\mathrm{SO}_{2} \mathrm{C}_{8} \mathrm{~F}_{17}\right)_{2}\right]_{4}$ & - + Catalyst for Bayer-Villiger oxidation & 153 \\
\hline $\mathrm{R}_{\mathrm{f} n} \mathrm{CH}_{2} \mathrm{CH}_{2} \mathrm{Li}_{/} \mathrm{Et}_{2} \mathrm{O}, n=6,8$ & $\begin{aligned} \Rightarrow & \mathrm{R}_{\mathrm{fn}}\left(\mathrm{CH}_{2}\right)_{2} \mathrm{I} /{ }^{\mathrm{t}} \mathrm{BuLi} \text {, ether; + } \mathrm{HSi}\left({ }^{\mathrm{i}} \mathrm{Pr}\right)_{2} \mathrm{Cl} \rightarrow \\
& \mathrm{R}_{\mathrm{ff}}\left(\mathrm{CH}_{2}\right)_{2} \mathrm{Si}\left({ }^{\mathrm{i}} \mathrm{Pr}\right)_{2} \mathrm{Hs}\end{aligned}$ & 143 \\
\hline $\mathrm{R}_{\mathrm{f} 8} \mathrm{CH}_{2} \mathrm{CH}_{2} \mathrm{ZnI} / \mathrm{THF}$ & $\Rightarrow \mathrm{R}_{\mathrm{fn}} \mathrm{CH}_{2} \mathrm{CH}_{2} \mathrm{I}+\mathrm{Zn} / \mathrm{THF}, \mathrm{BrCH}_{2} \mathrm{CH}_{2} \mathrm{Br},\left(\mathrm{CH}_{3}\right)_{3} \mathrm{CCl} \rightarrow$ & 131 \\
\hline $\mathrm{R}_{\mathrm{f} 6} \mathrm{CH}_{2} \mathrm{CH}_{2} \mathrm{MgI}$ & $\begin{array}{l}\text { - Precursor for the synthesis of the first fluorous tin } \\
\text { hydride/azide: }\left\{\left(\mathrm{R}_{\mathrm{f} 6} \mathrm{CH}_{2} \mathrm{CH}_{2}\right) \mathrm{SnX}, \mathrm{X}=\mathrm{H}, \mathrm{N}_{3}\right\} \\
\text { applied in "Fluorous Synthesis" }\end{array}$ & 151,154 \\
\hline
\end{tabular}


Tab. 9.2-7. Fluorous aldehydes and ketones

\begin{tabular}{|c|c|c|}
\hline Compound $[C, F, H, O]$ & Remarks (" $\Rightarrow$ " for synthesis of; “•+" for reaction of; etc.) & Ref. \\
\hline $\mathrm{C}_{8} \mathrm{~F}_{17} \mathrm{CHO}$ & $\Rightarrow \mathrm{C}_{8} \mathrm{~F}_{17} \mathrm{I}+\left(\mathrm{CH}_{3}\right)_{2} \mathrm{NCHO} / \mathrm{Al}-\mathrm{PbBr}_{2}$, r.t. $\rightarrow$ yield: $95 \%$ & 155 \\
\hline $\mathrm{R}_{\mathrm{fn}} \mathrm{CHO}, n=1,2,3,7$ & $\begin{aligned} \Rightarrow & \mathrm{R}_{\mathrm{fn}} \mathrm{CO}_{2} \mathrm{R}+\mathrm{LiAlH}_{4} / \text { ether, }-78^{\circ} \mathrm{C}, \text { "inverse addition" } \rightarrow \\
& 70-76 \%\end{aligned}$ & 156 \\
\hline $\mathrm{R}_{\mathrm{fn}} \mathrm{CH}_{2} \mathrm{CHO}$ & $\begin{aligned} \Rightarrow & \mathrm{R}_{\mathrm{fn}} \mathrm{I}+\mathrm{CH}_{2}=\mathrm{CHOAc} / \mathrm{AIBN}, 4 \mathrm{~h}, 80{ }^{\circ} \mathrm{C} \rightarrow \mathrm{R}_{\mathrm{fn}} \mathrm{CH}_{2} \mathrm{CHIOAc} ; \\
& +\mathrm{CH}_{2}=\mathrm{CHCO}_{2} \mathrm{H} / \mathrm{C}_{2} \mathrm{H}_{2} \mathrm{Cl}_{4} \text {, reflux, } 12 \mathrm{~h} \rightarrow \text { aldehyde yield: } \\
& 85 \%\end{aligned}$ & 157 \\
\hline $\mathrm{R}_{\mathrm{f} 6} \mathrm{CH}\left(\mathrm{CH}_{3}\right) \mathrm{CHO}$ & $\begin{aligned} \Rightarrow & \mathrm{R}_{\mathrm{f} 6} \mathrm{CH}=\mathrm{CH}_{2}+\mathrm{CO} / \mathrm{H}_{2} \text {-cat } \rightarrow 78 \% \text { ee at } 21 \% \text { conversion; } \\
& \text { cat: a polymer supported }(R, S) \text {-BINAPHOS-Rh }(\mathrm{I}) \text { complex }\end{aligned}$ & 158 \\
\hline $\mathrm{C}_{n} \mathrm{~F}_{2 n+1}\left(\mathrm{CH}_{2}\right)_{m} \mathrm{CHO}, m=1-4$ & $\begin{array}{l}\Rightarrow \text { Oxidation of } \mathrm{C}_{n} \mathrm{~F}_{2 n+1}\left(\mathrm{CH}_{2}\right)_{m} \mathrm{CH}_{2} \mathrm{OH} \text { by (a), (b) or } \\
\text { (c) methods: (a) Swern, (b) } \mathrm{PyH}^{+} \mathrm{CrO}_{3} \mathrm{Cl}^{-} \text {, (c) Dess-Martin } \\
\text { periodinane }\end{array}$ & 159,126 \\
\hline $3,5-\left(\mathrm{C}_{8} \mathrm{~F}_{17}\right)_{2} \mathrm{C}_{6} \mathrm{H}_{3} \mathrm{CHO}$ & $\begin{array}{l}\Rightarrow 3,5-\left(\mathrm{R}_{\mathrm{f} 8}\right)_{2} \mathrm{C}_{6} \mathrm{H}_{3} \mathrm{CH}_{2} \mathrm{OH}+\mathrm{PyH}^{+} \mathrm{CrO}_{3} \mathrm{Cl}^{-} / \mathrm{CH}_{2} \mathrm{Cl}_{2} \rightarrow \\
\text { yield: } 85 \%\end{array}$ & 68 \\
\hline $\mathrm{R}_{\mathrm{fn}} \mathrm{COCH}_{3}, \mathrm{R}_{\mathrm{fn}} \mathrm{COCH}_{2} \mathrm{COR}_{\mathrm{f} m}$ & $\begin{array}{l}\Rightarrow \mathrm{R}_{\mathrm{fn}} \mathrm{CO}_{2} \mathrm{H}+\mathrm{CH}_{3} \mathrm{MgBr} \rightarrow \mathrm{R}_{\mathrm{fn}} \mathrm{COCH}_{3} ;+\mathrm{R}_{\mathrm{f} m} \mathrm{CO}_{2} \mathrm{C}_{2} \mathrm{H}_{5}, \\
\quad \mathrm{NaOC}_{2} \mathrm{H}_{5} \rightarrow \text { good overall yields: (a) } n=m=6, \\
\quad \text { (b) } n, m=6,1 ; n=m=6,7\end{array}$ & 160 \\
\hline $\mathrm{R}_{\mathrm{f} 8} \mathrm{CH}_{2} \mathrm{CH}_{2} \mathrm{COCF}_{3}$ & $\Rightarrow \mathrm{R}_{\mathrm{f} 8} \mathrm{CH}_{2} \mathrm{CH}_{2} \mathrm{I}+\mathrm{Mg} /$ ether, $\mathrm{CF}_{3} \mathrm{CO}_{2} \mathrm{CH}_{3} \rightarrow$ yield: $32 \%$ & 161 \\
\hline $\begin{array}{l}\mathrm{F}\left[\mathrm{CF}\left(\mathrm{CF}_{3}\right) \mathrm{CF}_{2} \mathrm{O}\right]_{n} \mathrm{CF}\left(\mathrm{CF}_{3}\right) \\
\quad \mathrm{COCH}_{2}-\mathrm{COCH}_{3}, n=1-4\end{array}$ & $\begin{aligned} \Rightarrow & \mathrm{F}\left[\mathrm{CF}\left(\mathrm{CF}_{3}\right) \mathrm{CF}_{2} \mathrm{O}\right]_{n} \mathrm{CF}\left(\mathrm{CF}_{3}\right) \mathrm{CO}_{2} \mathrm{CH}_{3}+\mathrm{CH}_{3} \mathrm{COCH}_{3} / \\
& \mathrm{NaH} \rightarrow \text { no yield data reported }\end{aligned}$ & 162 \\
\hline
\end{tabular}


Tab. 9.2-8. Fluorous carboxylic acids

\begin{tabular}{|c|c|c|}
\hline Compound $[C, F, H, O]$ & Remarks (“" $\Rightarrow$ " for synthesis of; “•+" for reaction of; etc.) & Ref. \\
\hline $\mathrm{R}_{\mathrm{fn}} \mathrm{CO}_{2} \mathrm{H}$ & $\begin{aligned} \Rightarrow & \mathrm{R}_{\mathrm{fn}} \mathrm{CF}_{2} \mathrm{I}+\mathrm{XSO}_{3} \mathrm{H}(\mathrm{X}=\mathrm{Cl}, \mathrm{F}) \rightarrow \mathrm{R}_{\mathrm{fn}} \mathrm{CF}_{2} \mathrm{OSO}_{2} \mathrm{X} \\
& +\mathrm{H}_{2} \mathrm{O} \rightarrow\end{aligned}$ & 163 \\
\hline $\mathrm{R}_{\mathrm{fn}} \mathrm{CO}_{2} \mathrm{H}, n=2,3,5,6,7,11$ & $\begin{array}{l}\Rightarrow \mathrm{R}_{\mathrm{fn} n+1} \mathrm{I}(\mathrm{Br})+\text { Rongalite- } \mathrm{NaHCO}_{3} / \mathrm{DMF}_{-} \mathrm{H}_{2} \mathrm{O} \rightarrow \\
\quad \text { yield: } 51-86 \%\end{array}$ & 164 \\
\hline $\mathrm{C}_{n} \mathrm{~F}_{2 n+1} \mathrm{CO}_{2} \mathrm{H}, n=4,6,8$ & $\begin{array}{l}\Rightarrow \mathrm{C}_{n} \mathrm{~F}_{2 n+1} \mathrm{I}+\mathrm{Zn}-\mathrm{Cu} / \mathrm{CO}_{2},\left(\mathrm{CH}_{3} \mathrm{O}\right)_{3} \mathrm{PO},-20^{\circ} \mathrm{C} \rightarrow \\
\quad \text { yield: } 91 \%\end{array}$ & 165 \\
\hline $\begin{array}{l}\left(\mathrm{CF}_{3}\right)_{2} \mathrm{CFOCF}\left(\mathrm{CF}_{3}\right) \mathrm{CO}_{2} \mathrm{H}(+)- \\
\quad \text { and }(-) \text {-PIPA }\end{array}$ & $\begin{array}{l}\Rightarrow(+)-\mathrm{PIPA} \text { and }(-)-\mathrm{PIPA} \text { have been prepared from } \\
\mathrm{CF}_{3} \mathrm{COCF}_{3} \text { and } \mathrm{CF}_{3} \mathrm{CFCF}_{2} \mathrm{O} \text { precursors by a } \\
\text { multistep synthesis }\end{array}$ & 166 \\
\hline $\mathrm{C}_{6} \mathrm{~F}_{13} \mathrm{CH}_{2} \mathrm{CO}_{2} \mathrm{H}$ & $\begin{array}{l}\Rightarrow \mathrm{R}_{\mathrm{f} 6}\left(\mathrm{CH}_{2}\right)_{2} \mathrm{OH}+\mathrm{CrO}_{3} / \mathrm{H}_{2} \mathrm{SO}_{4} \text {, acetone-ether } \rightarrow \\
\quad \text { yield: } 98 \% \\
\text { - }+\mathrm{Rh}_{2}(\mathrm{OAc})_{4} / \text { toluene, } 110^{\circ} \mathrm{C}(-4 \mathrm{AcOH}) \rightarrow \\
\quad\left(\mathrm{R}_{\mathrm{f} 6} \mathrm{CH}_{2} \mathrm{CO}_{2}\right)_{4} \mathrm{Rh}_{2}\end{array}$ & 167,168 \\
\hline$\left(\mathrm{CF}_{3}\right)_{3} \mathrm{COCH}_{2} \mathrm{CO}_{2} \mathrm{H}$ & $\begin{aligned} \Rightarrow & \left(\mathrm{CF}_{3}\right)_{2} \mathrm{CCF}_{2} \mathrm{O} \\
& \mathrm{ClCH}_{2} \mathrm{CO}_{2} \mathrm{R} \rightarrow\end{aligned}$ & 169 \\
\hline $\begin{array}{c}\mathrm{R}_{\mathrm{fn}}\left(\mathrm{CH}_{2}\right)_{m} \mathrm{OCH}_{2} \mathrm{CO}_{2} \mathrm{H} \\
\quad n / m=7 / 1,8 / 2,10 / 2\end{array}$ & $\begin{array}{l}\Rightarrow \mathrm{R}_{\mathrm{fn}}\left(\mathrm{CH}_{2}\right)_{m} \mathrm{OH}+\mathrm{BrCH}_{2} \mathrm{CO}_{2} \mathrm{H} / \mathrm{NaH}, \mathrm{THF}, \text { r.t. } \rightarrow \\
\quad \text { yield: } 70-96 \% \\
\bullet+\mathrm{BH}_{3} / \mathrm{THF}, 0{ }^{\circ} \mathrm{C} \text { to r.t. } \rightarrow \mathrm{R}_{\mathrm{fn}}\left(\mathrm{CH}_{2}\right)_{m} \mathrm{O}\left(\mathrm{CH}_{2}\right)_{2} \mathrm{OH}, \\
\quad \text { yield: } 79-96 \%\end{array}$ & 170 \\
\hline $\mathrm{R}_{\mathrm{fn}} \mathrm{CH}_{2} \mathrm{CH}_{2} \mathrm{CO}_{2} \mathrm{H}, n=6,8,10$ & $\begin{aligned} \Rightarrow & \mathrm{R}_{\mathrm{fn}} \mathrm{CH}_{2} \mathrm{CH}_{2} \mathrm{I}+\mathrm{Mg} / \text { ether } \rightarrow \mathrm{R}_{\mathrm{fn}} \mathrm{CH}_{2} \mathrm{CH}_{2} \mathrm{MgI} ;+ \\
& \mathrm{CO}_{2} \rightarrow \\
\Rightarrow & \mathrm{R}_{\mathrm{f} 6}\left(\mathrm{CH}_{2}\right)_{3} \mathrm{OH}+\mathrm{NaIO}_{4} \text {, cat-RuCl}{ }_{3} \rightarrow \text { yield: } 64 \% \\
\bullet & +\mathrm{SOCl}_{2} \rightarrow \mathrm{R}_{\mathrm{f} 6}\left(\mathrm{CH}_{2}\right)_{2} \mathrm{COCl} ;+ \text { ferrocene } / \mathrm{AlCl}_{3} \rightarrow \\
& -\left(\mathrm{R}_{\mathrm{f}}-\mathrm{acyl}\right)_{1,2} \mathrm{Fc}\end{aligned}$ & 171 \\
\hline$\left(\mathrm{C}_{8} \mathrm{~F}_{17} \mathrm{CH}_{2} \mathrm{CH}_{2} \mathrm{CH}_{2}\right)_{2} \mathrm{CH}_{2} \mathrm{CO}_{2} \mathrm{H}$ & $\begin{array}{l}\Rightarrow \mathrm{R}_{\mathrm{f} 8}\left(\mathrm{CH}_{2}\right)_{3} \mathrm{I}+\mathrm{CH}_{2}\left(\mathrm{CO}_{2} \mathrm{C}_{2} \mathrm{H}_{5}\right)_{2} \rightarrow \rightarrow \rightarrow \text { overall } \\
\quad \text { yield: } 58 \%\end{array}$ & 172 \\
\hline $\begin{array}{l}\mathrm{C}_{8} \mathrm{~F}_{17} \mathrm{CH}_{2} \mathrm{CH}_{2} \mathrm{CON}\left(\mathrm{CH}_{2} \mathrm{CH}_{2} \mathrm{CH}_{2}-\right. \\
\left.\quad \mathrm{C}_{8} \mathrm{~F}_{17}\right) \mathrm{CH}_{2} \mathrm{CH}_{2} \mathrm{CO}_{2} \mathrm{H}\end{array}$ & $\begin{array}{l}\Rightarrow \text { Used as a protective group for oligosaccharide } \\
\text { synthesis } \\
\text { - Precursor for new fluorous supports for peptide } \\
\text { synthesis }\end{array}$ & 173 \\
\hline $3,4,5-\left[\mathrm{C}_{8} \mathrm{~F}_{17}\left(\mathrm{CH}_{2}\right)_{4} \mathrm{O}\right]_{3} \mathrm{C}_{6} \mathrm{H}_{2} \mathrm{CO}_{2} \mathrm{H}$ & $\begin{array}{l}\Rightarrow \text { Preparation and use for Mitsunobu-inversion of } \\
\text { chiral sec-alcohols }\end{array}$ & 174 \\
\hline
\end{tabular}


Tab. 9.2-9. Fluorous esters and carbonic acid derivatives

\begin{tabular}{|c|c|c|}
\hline Compound $[C, F, H, O]$ & $\begin{array}{l}\text { Remarks (“ } \Rightarrow \text { " for synthesis of; “ } \bullet+" \text { for } \\
\text { reaction of; etc.) }\end{array}$ & Ref. \\
\hline $\mathrm{R}_{\mathrm{fn}} \mathrm{CO}_{2} \mathrm{C}_{2} \mathrm{H}_{5}, n=4,6,8$ & $\begin{array}{l}\Rightarrow \mathrm{R}_{\mathrm{fn}} \mathrm{I}+\mathrm{ClCO}_{2} \mathrm{C}_{2} \mathrm{H}_{5}+\left(\mathrm{C}_{2} \mathrm{H}_{5} \mathrm{O}\right)_{2} \mathrm{CO}+\mathrm{Zn}-\mathrm{Cu} \rightarrow \\
\quad \text { yield: } 50-70 \%\end{array}$ & 175 \\
\hline $\mathrm{Br}\left(\mathrm{CF}_{2}\right)_{6} \mathrm{CO}_{2} \mathrm{C}_{2} \mathrm{H}_{5}$ & $\begin{array}{l}\Rightarrow \underset{67 \%}{\mathrm{Br}\left(\mathrm{CF}_{2}\right)_{6} \mathrm{CCl}_{3}+\mathrm{SO}_{3} / \mathrm{H}_{2} \mathrm{SO}_{4} ;+\mathrm{C}_{2} \mathrm{H}_{5} \mathrm{OH} \rightarrow \text { yield: }}\end{array}$ & 176 \\
\hline $\mathrm{C}_{3} \mathrm{~F}_{7} \mathrm{OCF}\left(\mathrm{CF}_{3}\right) \mathrm{CF}_{2} \mathrm{OCF}\left(\mathrm{CF}_{3}\right) \mathrm{CO}_{2} \mathrm{CH}_{3}$ & $\begin{aligned} \Rightarrow & \mathrm{C}_{3} \mathrm{~F}_{7} \mathrm{OCF}\left(\mathrm{CF}_{3}\right) \mathrm{CF}_{2} \mathrm{OCF}\left(\mathrm{CF}_{3}\right) \mathrm{COF}+\mathrm{CH}_{3} \mathrm{OH} / \mathrm{Na}_{2} \mathrm{CO}_{3}, \\
& \mathrm{MgSO}_{4} \text {, silica gel } \rightarrow \text { yield: } 86 \%\end{aligned}$ & 85 \\
\hline $\mathrm{R}_{\mathrm{f} 8} \mathrm{CH}_{2} \mathrm{CH}_{2} \mathrm{CH}\left(\mathrm{CO}_{2} \mathrm{CH}_{3}\right)_{2}$ & $\begin{aligned} \Rightarrow & \mathrm{R}_{\mathrm{f} 8} \mathrm{CH}_{2} \mathrm{CH}_{2} \mathrm{I}+\mathrm{CH}_{2}\left(\mathrm{CO}_{2} \mathrm{CH}_{3}\right)_{2} / \mathrm{NaH} \text { or } \mathrm{K}_{2} \mathrm{CO}_{3} \text { in } \\
& \mathrm{THF} \rightarrow \text { high yields }(\sim 85 \%) \text { with negligible } \\
& \text { elimination and dialkylation }\end{aligned}$ & 177 \\
\hline $\mathrm{R}_{\mathrm{f} 8}\left(\mathrm{CH}_{2}\right)_{n} \mathrm{CO}_{2} \mathrm{CH}_{2} \mathrm{CF}_{3}, n=1,2$ & $\begin{aligned} \Rightarrow & \mathrm{R}_{\mathrm{f} 8} \mathrm{CH}_{2} \mathrm{CH}_{2} \mathrm{OH} \rightarrow \sim \mathrm{CH}_{2} \mathrm{OTs} \rightarrow \sim \mathrm{CH}_{2} \mathrm{Br} \rightarrow \\
& \sim \mathrm{CH}_{2} \mathrm{MgBr} \rightarrow \sim \mathrm{CH}_{2} \mathrm{CO}_{2} \mathrm{H} \rightarrow \sim \mathrm{CH}_{2} \mathrm{COCl} \rightarrow \\
& \text { title ester, overall yield: } 55 \%\end{aligned}$ & 178 \\
\hline$\left(\mathrm{CF}_{3}\right)_{3} \mathrm{CCO}_{2} \mathrm{C}_{2} \mathrm{H}_{5}$ & $\begin{aligned} \Rightarrow & \left(\mathrm{CF}_{3}\right)_{2} \mathrm{C}=\mathrm{CF}_{2} / \mathrm{CsF}+\mathrm{ClC}(\mathrm{O}) \mathrm{OC}_{2} \mathrm{H}_{5} \rightarrow \text { yield: } 76 \% \\
& \mathrm{bp}=106{ }^{\circ} \mathrm{C}\end{aligned}$ & 179 \\
\hline 4- $\mathrm{R}_{\mathrm{f} 8} \mathrm{CH}_{2} \mathrm{CH}_{2} \mathrm{C}_{6} \mathrm{H}_{4} \mathrm{CO}_{2} \mathrm{CH}_{3}$ & $\begin{aligned} \Rightarrow & 4-\mathrm{BrC}_{6} \mathrm{H}_{4} \mathrm{CO}_{2} \mathrm{CH}_{3}+\mathrm{R}_{\mathrm{f} 8} \mathrm{CH}=\mathrm{CH}_{2} / \text { Pd-cat, } \mathrm{NaOAc}, \\
& \mathrm{DMF}, 125{ }^{\circ} \mathrm{C}, 14 \mathrm{~h}, \rightarrow \mathrm{R}_{\mathrm{f} 8} \mathrm{CH}=\mathrm{CH}_{2} \mathrm{C}_{6} \mathrm{H}_{4} \mathrm{CO}_{2} \mathrm{CH}_{3} \\
& (72 \%) ;+\mathrm{H}_{2} / \mathrm{Pd}-\mathrm{C} \rightarrow(88 \%)\end{aligned}$ & 180 \\
\hline$\left(\mathrm{CF}_{3}\right)_{3} \mathrm{CCH}_{2} \mathrm{CH}_{2} \mathrm{CO}_{2} \mathrm{CH}_{3}$ & $\begin{array}{l}\Rightarrow\left(\mathrm{CF}_{3}\right)_{3} \mathrm{CH}+\mathrm{CH}_{2}=\mathrm{CHCO}_{2} \mathrm{CH}_{3} /\left(\mathrm{C}_{2} \mathrm{H}_{5}\right)_{3} \mathrm{~N} \rightarrow \\
\quad \text { yield: } 30 \%\end{array}$ & 181 \\
\hline $3,5-\left(\mathrm{C}_{8} \mathrm{~F}_{17}\right)_{2} \mathrm{C}_{6} \mathrm{H}_{3} \mathrm{CO}_{2} \mathrm{CH}_{3}$ & $\begin{aligned} \Rightarrow & \mathrm{R}_{\mathrm{f} 8} \mathrm{I}+3,5-\mathrm{Br}_{2} \mathrm{C}_{6} \mathrm{H}_{3} \mathrm{CO}_{2} \mathrm{CH}_{3}+\mathrm{Cu} / \mathrm{DMSO} \\
& 135^{\circ} \mathrm{C} \rightarrow \text { yield: } 79 \%\end{aligned}$ & 68 \\
\hline $\mathrm{R}_{\mathrm{f} 6} \mathrm{CH}_{2} \mathrm{CH}_{2} \mathrm{OCOCl}$ & $\begin{array}{l}\Rightarrow \mathrm{R}_{\mathrm{fn}} \mathrm{CH}_{2} \mathrm{CH}_{2} \mathrm{OH}+\mathrm{COCl}_{2} / \text { toluene, reflux } 24 \mathrm{~h} \rightarrow \\
\text { yield: } \sim 95 \%\end{array}$ & 182 \\
\hline$\left(\mathrm{CF}_{3}\right)_{3} \mathrm{COCOCl}$ & $\Rightarrow\left(\mathrm{CF}_{3}\right)_{3} \mathrm{COK}+\mathrm{COCl}_{2} /$ mesitylene $\rightarrow$ yield: $54 \%$ & 183 \\
\hline $\mathrm{R}_{\mathrm{f}} \mathrm{CH}_{2} \mathrm{NCO}$ & $\begin{aligned} \Rightarrow & \mathrm{R}_{\mathrm{f} 7} \mathrm{CH}_{2} \mathrm{NH}_{2}+\mathrm{COCl}_{2} \rightarrow \text { yield: } 78 \%, \mathrm{bp}= \\
& 166-168{ }^{\circ} \mathrm{C}\end{aligned}$ & 42 \\
\hline $\mathrm{R}_{\mathrm{f} 9} \mathrm{CH}_{2} \mathrm{NCO}$ & $\Rightarrow \mathrm{R}_{\mathrm{f} 9} \mathrm{CH}_{2} \mathrm{NH}_{2}+\mathrm{COCl}_{2} /$ dioxane $\rightarrow \mathrm{mp}=46-47^{\circ} \mathrm{C}$ & 184 \\
\hline$\left(\mathrm{C}_{7} \mathrm{~F}_{15} \mathrm{CH}_{2}\right)_{2} \mathrm{NCOCl}$ & $\begin{aligned} \Rightarrow & \left(\mathrm{C}_{7} \mathrm{~F}_{15} \mathrm{CH}_{2}\right)_{2} \mathrm{NH}+\text { excess } \mathrm{COCl}_{2} / \text { autogenous } \\
& \text { pressure, } 140{ }^{\circ} \mathrm{C}, 14 \mathrm{~h} \rightarrow \text { yield: } 100 \%, \mathrm{mp}=22{ }^{\circ} \mathrm{C}, \\
& \text { bp }=70{ }^{\circ} \mathrm{C} / 0.1 \mathrm{mmHg}\end{aligned}$ & 119 \\
\hline $\begin{array}{l}\mathrm{R}_{\mathrm{fn}} \mathrm{CH}_{2} \mathrm{CH}_{2} \mathrm{~N}=\mathrm{C}=\mathrm{NCH}_{2} \mathrm{CH}_{2} \mathrm{R}_{\mathrm{fn}}, \\
\quad n=4,6,8\end{array}$ & $\begin{aligned} \Rightarrow & \mathrm{R}_{\mathrm{fn}}\left(\mathrm{CH}_{2}\right)_{2} \mathrm{~N}_{3}+\mathrm{PPh}_{3} / \mathrm{THF},<\text { r.t. } \rightarrow \\
& \mathrm{R}_{\mathrm{fn}}\left(\mathrm{CH}_{2}\right)_{2} \mathrm{~N}=\mathrm{PPh}_{3} ;+\mathrm{CS}_{2} \rightarrow \\
\Rightarrow & {\left[\mathrm{R}_{\mathrm{fn}}\left(\mathrm{CH}_{2}\right)_{2} \mathrm{NH}\right]_{2} \mathrm{CO}+\mathrm{Ph}_{3} \mathrm{PBr}_{2}+\mathrm{N}\left(\mathrm{C}_{2} \mathrm{H}_{5}\right)_{3} \text { in } } \\
& \mathrm{CH}_{2} \mathrm{Cl}_{2} / \mathrm{C}_{6} \mathrm{~F}_{14} \rightarrow\end{aligned}$ & 185,186 \\
\hline $\begin{array}{l}\mathrm{R}_{\mathrm{fn}}\left(\mathrm{CH}_{2}\right)_{m} \mathrm{O}_{2} \mathrm{CNHNHCO}_{2}\left(\mathrm{CH}_{2}\right)_{m} \mathrm{R}_{\mathrm{fn}} \\
\quad n / m=3 / 1,4 / 2,6 / 2,6 / 3\end{array}$ & $\begin{array}{l}\text { - }+\mathrm{NBS} \text { or } \mathrm{Br}_{2} / \mathrm{py}, \mathrm{CH}_{2} \mathrm{Cl}_{2} \rightarrow \\
\quad \mathrm{R}_{\mathrm{fn}}\left(\mathrm{CH}_{2}\right)_{m} \mathrm{O}_{2} \mathrm{CN}=\mathrm{NCO}_{2}\left(\mathrm{CH}_{2}\right)_{m} \mathrm{R}_{\mathrm{fn}} ; \text { yield: } 79-100 \% \\
\quad \text { used for fluorous Mitsunobu reaction }\end{array}$ & 182,187 \\
\hline
\end{tabular}




\section{References}

1 (a) Grosse, A. V.; Cady, G. H. 'Properties of Fluorocarbons'. Ind. Eng. Chem. 1947, 39, 367-374. (b) For a historic disclosure of war time developments in fluorine chemistry, see: ibid. pp. 236-434.

2 SAndford, G. Tetrahedron 2003, 59, 437454.

3 Imeda, I.; Watanabe, H.; Hirao, T.; Kurosawa, H. J. Fluorine Chem. 1994, 69, 97-102.

4 Zapevalov, A. Ya.; Plashkin, V. S.; Kolenko, I. P.; Neifel'd, P. G. Zh. Org. Khim. 1977, 13, 2504-2507.

5 LaZerte, J. D.; Hals, L. J.; Reid, T. S.; Sмітн, G. H. J. Am. Chem. Soc. 1953, 75, 4525-4528.

6 Synthesis of Fluoroorganic Compounds, Knunyants, I. L.; JaKobson, G. G., Eds.; Springer-Verlag: Berlin 1985; pp. 9-10.

7 Sanchez, V.; Greiner, J. Tetrahedron Lett. 1993, 34, 2931-2932.

8 Paskovich, D.; Gaspar, P.; Hammond, G. S. J. Org. Chem. 1967, 32, 833-834.

9 (a) Haszeldine, R. N. J. Chem. Soc. 1953, 3761-3768. (b) Selective photochemical synthesis of $\mathrm{R}_{\mathrm{fn}} \mathrm{Br} / \mathrm{I}$, see: ZHANG, L.; ZHANG, J.; YANG, W.; WANG, Y.; Fuss, W.; Weizbauer, S. J. Fluorine Chem. 1998, 88, 153-168.

10 Deev, L. E.; Nazarenko, T. I.; Pashrevich, K. I.; Ponomarev, V. G. Russ. Chem. Rev. 1992, 61, 40-54; Uspekhi Khimii 1992, 61, 75-101.

11 Chambers, R. D.; Hutchinson, J.; Mobbs, R. H.; Musgrave, W. K. R. Tetrahedron 1964, 20, 497-506.

12 Chen, Q.-Y.; He, Y.-B.; YANG, Z.-Y. J. Fluorine Chem. 1986, 34, 255-258.

13 Signé, E.; Blancou, H.; Commeyras, A. J. Fluorine Chem. 1995, 70, 197-200.

14 Hu, C.-M.; Quing, F.-L. J. Org. Chem. 1991, 56, 6348-6351.

15 Grondin, J.; Blancou, H.; Commeyras, A. J. Fluorine Chem. 1989, 45, 349-354.

16 Eapen, K. C.; Eisentraut, K. J.; Ryan, M. T.; ТАмвоRsкi, C. J. Fluorine Chem. 1986, 31, 405-416.

17 Mazalova, Z. I.; Sokolov, S. V.; Mazalov, S. A. Zh. Obshch. Khim. 1966, 36, 164-165.

18 Fukaya, H.; Hayashi, E.; Hayakawa, Y.; Аве, T. J. Fluorine Chem. 1997, 83, 117123.
19 Paciorek, K. J. L.; Masuda, S. R.; Shih, J. G.; Nakahara, J. H. J. Fluorine Chem. 1991, 53, 233-248.

20 Chen, G. J.; Chen, L. S.; Eapen, K. C. J. Fluorine Chem. 1993, 65, 59-66.

21 (a) Evans, F. W.; Litt, M. H.; WeidlerKubanek, A. M.; Avonda, F. P. J. Org. Chem. 1968, 33, 1839-1844. (b) Anello, L. G.; Sweeney, R. F. US Patent 3,409,512 (Allied Chemical Corporation), November 5, 1968 (application: September 27, 1966); Chem. Abstr. 1969, 70, 37177j.

22 Bekker, R. A.; Dyatkin, B. L.; Knunyants, I. L. Izv. Akad. Nauk SSSR Ser. Khim. 1970, 2738-2741.

23 Terjeson, R. J.; Renn, J.; Winter, R.; Gard, G. L. J. Fluorine Chem. 1997, 82, 73-78.

24 Terjeson, R. J.; Gard, G. L. J. Fluorine Chem. 1987, 35, 653-662.

25 Case, J. R.; Ray, N. H.; Roberts, H. L. J. Chem. Soc. 1961, 2070-2075.

26 Lagow, R. J.; Eujen, R.; Gerchman, L. L.; Morrison, J. A. J. Am. Chem. Soc. 1978, 100, 1722-1726.

27 Tiers, G. V. D. J. Am. Chem. Soc. 1955, 77, 4837-4838.

28 Tiers, G. V. D. J. Am. Chem. Soc. 1955 , 77, 6703-6704.

29 Smith, R. D.; Fawcett, F. S.; Coffman, D. D. J. Am. Chem. Soc. 1962, 84, 42854288.

30 Seyferth, D.; Tronich, W.; Smith, W. E.; Hopper, S. P. J. Organometal. Chem. 1974, 67, 341-352.

31 Haywood, L.; McKee, S.; Middletown, W. J. J. Fluorine Chem. 1991, 51, 419-431.

32 (a) Ishikawa, N.; Sasabe, M. J. Fluorine Chem. 1984, 25, 241-254; (b) Paleta, O.; Církva, V.; Kvíčala, J. J. Fluorine Chem. 1996, 80, 125-134.

33 Petrov, V. A.; Swearingen, S.; Hong, W.; Petersen, W. C. J. Fluorine Chem. 2001, 109, 25-31.

34 Tiers, G. V. D. J. Org. Chem. 1961, 26, 3515-3516.

35 Kamigata, N.; Fukushima, T.; Yoshida, M. Chem. Lett. 1990, 649-650.

36 Meussdoerffer, J. N.; Niederpruem, H.; Wechsberg, M. Ger. Offen DE 2,238,152 (BAYER A.-G.), February 14, 1974 (Application: August 3, 1972); Chem. Abstr. 1974, 80, 120258p. 
37 Tiers, G. V. D. J. Org. Chem. 1963, 28, 1244-1246.

38 Tiers, G. V. D. J. Fluorine Chem. 1998, 90, 49-51.

39 Zнu, D.-W. Synthesis 1993, 953-954.

40 Banks, R. E.; Cheng, W. M.; Haszeldine, R. N. J. Chem. Soc. 1962, 3407-3416.

41 Petrov, V. A.; Belen'kit, G. G.; German, L. S. Izv. Akad. Nauk SSSR Ser. Khim. 1985, 8, 1934-1935.

42 Sander, M. Monatsh. Chem. 1964, 95, 608-616.

43 Paciorek, K. J. L.; Nakahara, J. H.; Kratzer, R. H. J. Fluorine Chem. 1985, 30, 241-250.

44 Chambers, W. J.; Tullock, C. W.; Coffman, D. D. J. Am. Chem. Soc. 1962, 84, 2337-2343.

45 Peterson, W. M., Jr.; Radell, J.; Washburne, S. S. J. Fluorine Chem. 1972/ 73, 2, 437-441.

46 (a) Denson, D. D.; Smith, C. F.; Tамвовsкi, C. J. Fluorine Chem. 1973/74, 3, 247-258. (b) Sмith, C. F.; Soloski, E. J.; ТАмвовsкi, C. J. Fluorine Chem. 1974, 4, 35-45.

47 Kvíčala, J.; Mouyrin, J.-C.; Paleta, O. J. Fluorine Chem. 2002, 113, 195200.

48 Sekiya, A.; Ishikawa, N. Chem. Lett. 1977, 81-84.

49 McLoughim, V. C. R.; Thrower, J. Tetrahedron 1969, 25, 5921-5940.

50 (a) Hein, J. E.; Hultin, P. G. Synlett 2003, 635-638. (b) For nucleophilic perfluoroalkylation using perfluoroalkyl lithiums, see: Uno, H.; SUZu Kib, H. Synlett 1993, 91-96.

51 Chambers, R. D.; Magron, C.; Sandford, G. J. Chem. Soc., Perkin Trans. 1, 1999, 283-290.

52 (a) Burton, D. J.; YAnG, Z.-Y. Tetrahedron 1992, 48, 189-275. (b) BuRTON, D. J.; YANG, Z.-Y.; Morken, P. A. Tetrahedron 1994, 50, 2993-3063.

53 Koppel, I. A.; Pihl, V.; Koppel, J.; Anvia, F.; TAFt, R. W. J. Am. Chem. Soc. 1994, 116, 8654-8657.

54 Napoli, M.; Conte, L.; Guerrato, A. J. Fluorine Chem. 2001, 110, 47-58.

55 Legros, J.; Crousse, B.; Bonnet-Delpon, D.; BÉguÉ, J.-P.; Maruta, M. Tetrahedron 2002, 58, 4067-4070.

56 Brace, N. O.; Marshall, L. W.; Pinson,
C. J.; van Wingerden, G. J. Org. Chem 1984, 49, 2361-2368.

57 SZlávik, Z.; TÁrKáNYI, G.; GömöRY, Á.; RÁbAI, J. Org. Lett. 2000, 2, 2347-2349.

58 Napoli, M.; Gambaretto, G. P. J. Fluorine Chem. 1997, 84, 101-102.

59 Delyagina, N. I.; Pervova, E. Ya.; Knunyants, I. L. Izv. Akad. Nauk SSSR Ser. Khim. 1972, 21, 376-380.

60 Dмowski, W.; Wozniacki, R. J. Fluorine Chem. 1987, 36, 385-394.

61 Tittle, B. British Patent 1,153,187 (Imperial Chemical Industries), Month day, 1969 (application: Month day, year); Chem. Abstr. 1969, 71, 38789t.

62 Gontar', A. F.; Byкhovskaya, E. G.; KNunYANTS, I. L. Izv. Akad. Nauk. SSSR Ser. Khim. 1975, 24, 2279-2282.

63 Riess, J. G. In: Fluorine Chemistry at the Millennium: Fascinated by Fluorine. Ed. R. E. BAN Ks, Elsevier: Amsterdam 2000; Chapter 23.

64 Calas, P.; Moreau, P.; Commeyras, A. J. Chem. Soc., Chem. Commun. 1982, 433434.

65 von Werner, K. German Patent 3,247,728 (Hoechst A.-G.), July 5, 1984 (application: December 23, 1982); Chem. Abstr. 1985, 102, 5889f.

66 Nakamura, T.; Koga, Y. J. Chem. Soc., Perkin Trans. 2, 1998, 659-662.

67 (a) No author specified. British Patent 840,725 (Minnesota Mining and Manufacturing Company) July 6, 1960 (application: August 12, 1955); Chem. Abstr. 1961, 55, 6496h. (b) Tiers, G. V. D. US Patent 3,281,426 (Minnesota Mining and Manufacturing Company), October 25, 1966 (application: August 12, 1955, and May 1, 1961); Chem. Abstr. 1967, 66, 66740k. (c) Tiers, G. V. D. J. Am. Chem. Soc. 1960, 82, 5513. (d) Brace, N. O. US Patent 3,271,441 (E. I. du Pont de Nemours and Company), September 6, 1966 (application: June 18, 1962); Chem. Abstr. 1967, 66, 2388y

68 Kiss, L. E.; Kövesdi, I.; RÁbAI, J. J Fluorine Chem. 2001, 108, 95-109.

69 Hodges, A. M.; Winter, R.; Mohtasham, J.; Bailey, P.; Gard, G. L. J. Fluorine Chem. 2001, 110, 1-4.

70 Shimizu, R.; Yoneda, E.; Fuchikami, T. Tetrahedron Lett. 1996, 37, 5557-5560.

71 YANG, G.-S.; XIE, X.-j.; ZhAO, G.; DinG, Y. J. Fluorine Chem. 1999, 98, 159-161. 
172 9 Getting Started in Synthesis: A Tabular Guide to Selected Monofunctional Fluorous Compounds

72 Chen, G. J.; TамвоRsкi, C. J. Fluorine Chem. 1989, 43, 207-228.

73 Chen, W.; Xu, L.; XiaO, J. Tetrahedron Lett. 2001, 42, 4275-4278.

74 Rocaboy, C.; Rutherford, D.; Bennett, B. L.; Gladysz, J. A. J. Phys. Org. Chem. 2000, 13, 1-8.

75 Tiers, G. V. D.; Brown, H. A.; Reid, T. S. J. Am. Chem. Soc. 1953, 75, 5978-5979.

76 Brace, N. O. US Patent 3,016,406 (E. I. du Pont de Nemours and Company), January 9, 1962 (application: September 21, 1960); Chem. Abstr. 1962, 57, 2078a.

77 Boechl, W. Neth. Appl. 6,506,069. November 15, 1965 (application: May 14, 1964); Chem. Abstr. 1966, 64, 17421c.

78 Vincent, J.-M.; Rabion, A.; Yachandra, V. K.; Fish, R. H. Angew. Chem., Int. Ed. Engl. 1997, 36, 2346-2349.

79 Alvey, L. J.; Meier, R.; Soós, T.; Bernatis, P.; Gladysz, J. A. Eur. J. Inorg. Chem. 2000, 9, 1975-1984.

80 Winter, R.; Nixon, P. G.; Terjeson, R. J.; Мohtasham, J.; Ноlcomb, N. R.; Grainger, D. W.; Graham, D.; Castner, D. G.; Gard, G. L. J. Fluorine Chem. 2002, 115, 107-113.

81 Cavazzini, M.; Manfredi, A.; Montanari, F.; Quici, S.; Pozzi, G. Eur. J. Org. Chem. 2001, 4639-4649.

82 Ishihara, K.; Kondo, S.; Yamamoto, H. Synlett 2001, 1371-1374.

83 Rocaboy, C.; Gladysz, J. A. Chem.-Eur. J. 2003, 9, 88-95.

84 Annunziata, R.; Benaglia, M.; Cinquini, M.; Cozzi, F.; Pozzi, G. Eur. J. Org. Chem. 2003, 1191-1197.

85 Paleta, O.; Paleček, J.; Michálek, J. J. Fluorine Chem. 2002, 114, 51-53.

86 Kocharyan, S. T.; Rokhlin, E. M.; Cheburkov, Yu. A.; Knunyants, I. L. Izv. Akad. Nauk SSSR Ser. Khim. 1966, 10, 1870.

87 SZlávik, Z.; TÁrkáNyi, G.; TARCZAY, G.; GöмöRY, Á.; RÁbAI, J. J. Fluorine Chem. 1999, 98, 83-87.

88 Read, R. W.; Zhang, C. Tetrahedron Lett. 2003, 44, 7045-7047.

89 Wipf, P.; Reeves, J. T. Tetrahedron Lett. 1999, 40, 5139-5142.

90 Luo, Z.; Williams, J.; Read, R. W.; Curran, D. P. J. Org. Chem. 2001, 66, 4261-4266.

91 Pardo, J.; Cobas, A.; Guitián, E.; CAstedo, L. Org. Lett. 2001, 3, 3711-3714.

92 Kotora, M.; Hájek, M.; AmÉduri, B.; Boutevin, B. J. Fluorine Chem. 1994, 68, 49-56.

93 Církva, V.; Böhm, S.; Paleta, O. J. Fluorine Chem. 2000, 102, 159-168.

94 Nakamura, Y.; Takeuchi, S.; OKumura, K.; Ohgo, Y. Tetrahedron 2001, 57, 55655572.

95 Dondy, B.; Doussot, P.; Portella, C. Synthesis 1992, 995-998.

96 Matsubara, H.; Yasuda, S.; Sugiyama, H.; Ryu, I.; Fujiı, Y.; KitA, K. Tetrahedron 2002, 58, 4071-4076.

97 Makarov, K. N.; Abroskina, T. N.; Cheburkov, Yu. A.; Knunyants, I. L. Izv. Akad. Nauk SSSR Ser. Khim. 1976, 4, 940-942.

98 Delyagina, N. I.; Dyatkin, B. L.; Knunyants, I. L. Zh. Org. Khim. 1974, 10, 935-941.

99 Takada, N.; Aвe, T.; Sekiya, A. J. Fluorine Chem. 1998, 92, 167-171.

100 Církva, V.; Améduri, B.; Boutevin, B.; Paleta, O. J. Fluorine Chem. 1997, 83, 151-158.

101 Harris, J. F.; Sheppard, W. A. J. Org. Chem. 1961, 26, 354-358.

102 Lantz, A. US Patent 4,845,300 (Societe Atochem), July 4, 1989 (application: January 11, 1988); Chem. Abstr. 1989, 110, 57118p.

103 RÁbai, J.; Bonto, A.-M.; MiedziÁk, M.; SzaBó, D. The First International Sibirian Workshop-Advanced Inorganic Fluorides 'INTERSIBFLUORINE-2003' April 2-4, 2003. Novosibirsk, Russia. Proceedings of ISIF-2003. pp. 198-201. ISBN 5-90168804-5 E.

104 Winter, R.; Nixon, P. G.; Gard, G. L.; RADFORD, D. H.; НоLCOMb, N. R.; Grainger, D. W. J. Fluorine Chem. 2001, 107, 23-30.

105 Billard, T.; Roques, N.; Langlois, B. R. J. Org. Chem. 1999, 64, 3813-3820.

106 Crich, D.; Neelamkavil, S. Org. Lett. 2002, 4, 4175-4177.

107 Magnier, E.; Tordeux, M.; Goumont, R.; Magder, K.; Wakselman, C. $J$. Fluorine Chem. 2003, in the press.

108 Rocaboy, C.; Gladysz, J. A. Tetrahedron 2002, 58, 4007-4014.

109 Popov, V. I.; Boiko, V. N.; Yagupolskit, L. M. J. Fluorine Chem. 1982, 21, 365370.

110 Ignat'ev, N. V.; Boiko, V. N.; 
YAGUPol'skit, L. M. Zh. Org. Khim. 1985, 21, 653.

111 Wakselman, C.; Tordeux, M. J. Org. Chem. 1985, 50, 4047-4051.

112 Crich, D.; Neelamkavil, S. J. Am. Chem. Soc. 2001, 123, 7449-7450.

113 Rábai, J.; Szabó, D.; Borbás, E. K.; Kövesi, I.; Kövesdi, I.; Csám PaI, A.; GömÖRY, Á.; PASHINNIK, V. E.; Shermolovich, Y. G. J. Fluorine Chem. 2002, 114, 199-207.

114 Sinou, D.; Maillard, D.; Pozzi, G. Eur. J. Org. Chem. 2002, 269-275.

115 De Campo, F.; Lastécouères, D.; Vincent, J.-M.; Verlhac, J.-B. J. Org. Chem. 1999, 64, 4969-4971.

116 Bonrath, W.; HaAs, A.; Hoppmann, E.; Netscher, T.; Pauling, H.; Schager, F.; Wildermann, A. Adv. Synth. Catal. 2002, 344, 37-39.

117 Betzemeier, B.; Lhermitte, F.; Knochel, P. Synlett 1999, 489-491.

118 ten Brink, G.-J.; Vis, J. M.; Arends, I. W. C. E.; Sheldon, R. A. Tetrahedron 2002, 58, 3977-3983.

119 Silverstein, R. M.; Rodin, J. O.; Mill, T.; Woolf, C. J. Chem. Eng. Data 1964, 9, 588-592.

120 Husted, D. R.; Ahlbrecht, A. H. US Patent 2,782,184 (Minnesota Mining and Manufacturing Company) February 19, 1957 (application: July 17, 1953); Chem. Abstr. 1957, 51, 11376.

121 Petrov, V. A. Tetrahedron Lett. 2000, 41, 6959-6963.

122 Kiss, L. E.; RÁbaI, J.; VARGA, L.; KöVesdi, I. Synlett 1998, 1243-1245.

123 Nishida, M.; Ono, T.; Aвe, T. J. Fluorine Chem. 2001, 110, 63-74.

124 Gontar', A. F.; Glotov, E. N.; Rybachev, A. A.; KNunYants, I. L. Izv. Akad. Nauk SSSR Ser. Khim. 1984, 8, 1874-1877.

125 Szönyi, F.; Guennouni, F.; Cambon, A. J. Fluorine Chem. 1991, 55, 85-92.

126 Rocaboy, C.; Bauer, W.; Gladysz, J. A. Eur. J. Org. Chem. 2000, 2621-2628.

127 Linclau, B.; Singh, A. K.; Curran, D. P. J. Org. Chem. 1999, 64, 2835-2842.

128 Szlávik, Z.; TÁrkányi, G.; Gömöry, Á.; Tarczay, G.; Rábai, J. J. Fluorine Chem. 2001, 108, 7-14.

129 Maayan, G.; Fish, R. H.; Neumann, R. Org. Lett. 2003, 5, 3547-3550.

130 Moreno-Mañas, M.; Pleixats, R.; Villarroya, S. Synlett. 1999, 1996-1998.
References $\mid 173$

131 Rocaboy, C.; Hampel, F.; Gladysz, J. A. J. Org. Chem. 2002, 67, 6863-6870.

132 Chen, G. J.; Tамвorski, C. J. Fluorine Chem. 1990, 46, 137-159.

133 Horváth, I. T.; RÁbAI, J. US Patent 5,463,082 (Exxon Research and Engineering Company), October 31, 1995 (application: July 8, 1993); Chem. Abstr. 1995, 123, 87349a.

134 Alvey, L. J.; Rutherford, D.; Juliette, J. J. J.; Gladysz, J. A. J. Org. Chem. 1998, 63, 6302-6308.

135 van den Broeke, J.; Lutz, M.; Kooljman, H.; Spek, A. L.; Deelman, B.-J.; van Koten, G. Organometallics 2001, 20, 2114-2117.

136 van den BRoeke, J.; DE Wolf, E.; Deelman, B.-J.; van Koten, G. Adv. Synth. Catal. 2003, 345, 625-635.

137 van den Broeke, J.; Deelman, B.-J.; van Koten, G. Tetrahedron Lett. 2001, 42, 8085-8087.

138 Krishnamurti, R.; Bellew, D. R.; Surya Prakash, G. K. J. Org. Chem. 1991, 56, 984-989.

139 Surya Prakash, G. K.; Yudin, A. K. Chem. Rev. 1997, 97, 757-786.

140 Huang, Y.; Chen, D.; Quing, F.-L. Tetrahedron 2003, 59, 7879-7886.

141 Curran, D. P.; Ferritto, R.; Hua, Y. Tetrahedron Lett. 1998, 39, 4937-4940.

142 Studer, A.; Jegrer, P.; Wipf, P.; Curran, D. P. J. Org. Chem. 1997, 62, 2917-2924.

143 Zang, Q.; Rivkin, A.; Curran, D. P. J. Am. Chem. Soc. 2002, 124, 5774-5781.

144 Richter, B.; de Wolf, E.; van Koten, G.; Defelman, B.-J. J. Org. Chem. 2000, 65, 3885-3893.

145 de Wolf, E.; Speets, E. A.; Deflman, B.-J.; van Koten, G. Organometallics 2001, 20, 3686-3690.

146 Konakahara, T.; Okada, S.-i.; FuruhaShi, J.-I.; Sugaya, J.; Monde, T.; Nakayama, N.; Nemoto, K. Y. F.; Kamiusu Ki, T. J. Fluorine Chem. 2000, 101, 39-43.

147 Beyou, E.; Babin, P.; Bennetau, B., Dunogues, J.; Teyssie, D.; Boileau, S. Tetrahedron Lett. 1995, 36, 1843-1844.

148 Jenkins, P. M.; Steele, A. M.; Tsang, S. C. Catalysis Commun. 2003, 4, 45-50.

149 Curran, D. P.; Luo, Z.; Degenkolb, P. Bioorg. Med. Chem. Lett. 1998, 8, 24032408.

150 Hoshino, M.; Degenkolb, P.; Curran, D. P. J. Org. Chem. 1997, 62, 8341-8349. 
151 Curran, D. P.; Hadida, S. J. Am. Chem. Soc. 1996, 118, 2531-2532.

152 Curran, D. P.; Hadida, S.; Kim, S.-Y. Tetrahedron 1999, 55, 8997-9006.

153 HaO, X.; YamazaKi, O.; Yoshida, A.; Nishiкido, J. Tetrahedron Lett. 2003, 44, 4977-4980.

154 Studer, A.; Hadida, S.; Ferritto, R.; Kim, S.-Y.; Jeger, P.; Wipf, P.; Curran, D. P. Science 1997, 275, 823-826.

$155 \mathrm{Hu}$, C.-M.; TANG, X.-Q. J. Fluorine Chem. 1993, 61, 217-222.

156 Pierce, O. R.; Kane, T. G. J. Am. Chem. Soc. 1953, 76, 300-301.

157 Laurent, P.; Blancou, H.; Commeyras, A. Tetrahedron Lett. 1992, 33, 2489-2492.

158 Shibahara, F.; Nozaki, K.; Hiyama, T. J. Am. Chem. Soc. 2003, 125, 85558560.

159 Lévêque, L.; Le Blanc, M.; Pastor, R. Tetrahedron Lett. 1998, 39, 8857-8860.

160 (a) Betzemeier, B.; Lhermitte, F.; KNOCHEL, P. Tetrahedron Lett. 1998, 39, 6667-6670. (b) Croxtall, B.; Fawcett, J.; Hope, E. G.; Stuart, A. M. J. Fluorine Chem. 2003, 119, 65-73.

161 Ohsaka, Y.; Kobayashi, T.; Kubo, M. US Patent 4,946,935 (Daikin Industries, Ltd.), August 7, 1990 (application: April 4, 1988); Chem. Abstr. 1989, 110, 115489y.

162 Keim, W.; Vogt, M.; Wasserscheid, P.; Dreißen-Hölscher, B. J. Mol. Catal. A: Chemical 1999, 139, 171-175.

163 Hauptschein, M.; Braid, M. J. Am. Chem. Soc. 1961, 83, 2500-2507.

164 Huang, B.-N.; HaAs, A.; Lieb, M. J. Fluorine Chem. 1987, 36, 49-62.

165 Bénéfice-Malouet, S.; Blancou, H.; Itier, J.; Commeyras, A. Synthesis 1991, 647-648.

166 Kawa, H.; Yamaguchi, F.; Ishikawa, N. Chem. Lett. 1982, 745-748.

167 Achilefu, S.; Mansuy, L.; Selve, C.; Thiebaut, S. J. Fluorine Chem. 1995, 70, 19-26.

168 Endres, A.; Maas, G. J. Organomet. Chem. 2002, 643-644, 174-180.

169 PavLiK, F. J. US Patent 3,668,233 (Minnesota Mining and Manufacturing Company), June 6, 1972 (application: October 30, 1962); Chem. Abstr. 1972, 77, 100830d.

170 Elshani, S.; Kobzar, E.; Bartsch, R. A. Tetrahedon 2000, 56, 3291-3301.
171 (a) Jouani, A. N.; SzÖnYi, F.; Cambon, A. J. Fluorine Chem. 1992, 56, 85-92. (b) HAZAFY, D.; Sobociková, M.; ŠTĚPniČKA, P.; Ludvík, J.; Kotora, M. J. Fluorine Chem. 2003, in the press.

172 Loiseau, J.; Fouquet, E.; Fish, R. H.; Vincent, J.-M.; Verlhac, J.-B. J. Fluorine Chem. 2001, 108, 195-197.

173 (a) Miura, T.; Inazu, T. Tetrahedron Lett. 2003, 44, 1819-1821. (b) Mizuno, M.; Goto, K.; Miura, T.; Hosaka, D.; Inazu, T. J. Chem. Soc., Chem. Commun. 2003, 972-973. (c) MiUra, T.; Goto, K.; Hosaka, D.; Inazu, T. Angew. Chem., Int. Ed. Engl. 2003, 42, 2047-2051.

174 Markowicz, M. W.; Dembinski, R. Org. Lett. 2002, 4, 3785-3787.

175 Benefice, S.; Blancou, H.; Commeyras, A. Tetrahedron 1984, 40, 1541-1544.

176 Кім, Y. K. J. Org. Chem. 1967, 32, 36733675.

177 Trabelsi, H.; SzÖnyi, F.; Geribaldi, S. J. Fluorine Chem. 2001, 107, 177-181.

178 Hungerhoff, B.; Sonnenschein, H.; Theil, F. J. Org. Chem. 2002, 67, 17811785.

179 Knunyants, I. L.; Zeifman, Yu. V.; LantSeva, L. T. Dokl. Akad. Nauk SSSR Ser. Khim. 1980, 254, 117-120.

180 Filippov, D. V.; van Zoelen, D. J.; Oldfield, S. P.; van der Marel, G. A.; Overkleeft, H. S.; Drijfhout, J. W.; van Воом, J. H. Tetrahedron Lett. 2002, 43, 7809-7812.

181 Knunyants, I. L.; Kocharyan, S. T.; Rohlin, E. M. Izv. Akad. Nauk SSSR Ser. Khim. 1966, 6, 1057-1062.

182 Dobbs, A. P.; McGregor-Johnson, C. Tetrahedron Lett. 2002, 43, 2807-2810.

183 HudlickÝ, M. J. Fluorine Chem. 1982, 20, 649-658.

184 ReId, T. S. US Patent 2,706,733

(Minnesota Mining and Manufacturing Company), April 19, 1955; Chem. Abstr. 1956, 50, 2661f.

185 Trabelsi, H.; Bollens, E.; Jouani, M. A.; Gaysinski, M.; SzÖnyi, F.; Cambon, A. Phosphorus, Sulfur Silicon Relat. Elem. 1994, 90, 185-192.

186 Palomo, C.; Aizpurua, J. M.; Loinaz, I.; Fernandez-Berridi, M. J.; Irusta, L. Org. Lett. 2001, 3, 2361-2364.

187 Dandapani, S.; Curran, D. P. Tetrahedron 2002, 58, 3855-3864. 